\title{
Design and optimization of a highly efficient optical multipass system for $\gamma$-ray beam production from electron laser beam Compton scattering
}

\author{
K. Dupraz, K. Cassou, N. Delerue, P. Fichot, A. Martens, A. Stocchi, A. Variola, and F. Zomer \\ LAL, Université Paris-Sud, CNRS/IN2P3, Orsay, Bâtiment 200, BP 34, 91898 Orsay cedex, France
}

A. Courjaud and E. Mottay

Amplitude Systèmes, 6 allée du Doyen Georges Brus, 33600 Pessac, France

F. Druon

Laboratoire Charles Fabry de l'Institut d'Optique, UMR 8501 CNRS, Université Paris Sud, 91127 Palaiseau, France

G. Gatti and A. Ghigo

INFN-LNF, Via Enrico Fermi 40, 00044 Frascati Rome, Italy

T. Hovsepian, J. Y. Riou, and F. Wang

Alsyom, Parc des Algorithmes, Bâtiment Aristote, 9 Avenue du Marais, 95100 Argenteuil, France

A. C. Mueller

National Institute for Nuclear and Particle Physics, CNRS, 3 rue Michel-Ange, 75016 Paris, France

L. Palumbo

Universita di Roma La Sapienza, Dipartimento Energetica, Via Antonio Scarpa, 14-00161 Rome, Italy

L. Serafini and P. Tomassini

INFN-MI, Via Celoria 16, 20133 Milano, Italy

(Received 31 December 2013; published 26 March 2014)

A new kind of nonresonant optical recirculator, dedicated to the production of $\gamma$ rays by means of Compton backscattering, is described. This novel instrument, inspired by optical multipass systems, has its design focused on high flux and very small spectral bandwidth of the $\gamma$-ray beam. It has been developed to fulfill the project specifications of the European Extreme Light Infrastructure "Nuclear Pillar," i.e., the Gamma Beam System. Our system allows a single high power laser pulse to recirculate 32 times synchronized on the radio frequency driving accelerating cavities for the electron beam. Namely, the polarization of the laser beam and crossing angle between laser and electrons are preserved all along the 32 passes. Moreover, optical aberrations are kept at a negligible level. The general tools developed for designing, optimizing, and aligning the system are described. A detailed simulation demonstrates the high efficiency of the device.

DOI: 10.1103/PhysRevSTAB.17.033501

PACS numbers: 42.79.-e, 07.85.Fv, 42.15.Eq

\section{INTRODUCTION}

Compton scattering between a relativistic electron bunch and a high power laser pulse is one of the most efficient techniques to generate a high brilliance $\gamma$-ray beam [1]. However, this physical process exhibits a small cross section: therefore the densities of laser photons and

\footnotetext{
*Corresponding author. dupraz@lal.in2p3.fr

Published by the American Physical Society under the terms of the Creative Commons Attribution 3.0 License. Further distribution of this work must maintain attribution to the author $(s)$ and the published article's title, journal citation, and DOI.
}

electrons in the collision point must be very high in order to generate a large number of $\gamma$-ray photons at each collisions, and the repetition rate of the collisions must be maximized in order to achieve high $\gamma$-ray fluxes. As extensively reported elsewhere [1-5] the phase space density of the electron beam and of the laser beam at the collision point are key issues for achieving high fluxes of gamma rays with very small bandwidths, as those typically requested by nuclear physics experiments and applications. In this article, we concentrate on the optical design of the interaction point (IP) of a Compton $\gamma$-ray machine which has been proposed for the European Extreme Light Infrastructure "Nuclear Pillar," i.e., the Gamma Beam System (ELI-NP-GBS) $[1,3,4,6]$ project. 
As a construction project it will be funded by the European Commission in order to build a research infrastructure in Romania. ELI-NP-GBS will be aimed at exploring the scientific potential of a high brilliance gamma and high spectral density ray beam system, in the field of nuclear physics, namely photonuclear induced reactions. The performance gain is such that many applications and nuclear physics studies not possible hitherto might be performed. The goal is to open a new field, "nuclear photonics," allowing inedited experiments in fundamental nuclear physics and advanced applications in the field of national security, nuclear waste composition identification, and nuclear medicine.

The main specifications of such a Compton $\gamma$-ray source are: photon energy tunable in the range $1-20 \mathrm{MeV}$, root mean square (rms) bandwidth smaller than $0.5 \%$ and time average spectral density (TASD) larger than $5000 \gamma /(\mathrm{s} \mathrm{eV})$, with source spot sizes smaller than $100 \mu \mathrm{m}$ and linear polarization of the $\gamma$-ray beam larger than $95 \%$. At last, the peak brilliance of the $\gamma$-ray beam is expected to be larger than $10^{20} \gamma /\left(\mathrm{s} \mathrm{mm}^{2} \operatorname{mrad}^{2} 0.1 \%\right)$.

To reach this goal, a linear accelerator (LINAC) which combines $\mathrm{S}$ and $\mathrm{C}$ band accelerating cavities with the electrons provided by a photoinjector has been proposed [6] in order to maximize the electron beam phase space density at the collision point. The linac will be operated in multibunch mode, with an electron beam made of trains of a few tens of bunches with a repetition rate of $100 \mathrm{~Hz}$ and bunch separation of about $15 \mathrm{~ns}$. An extensive description of the electron linac and of the collimation system needed to monochromatize the $\gamma$-ray beam is described in [7].

To optimize the $\gamma$-ray beam quality, an optical system preserving the spectral bandwidth while maximizing the flux must be provided at the Compton IP. The presentation of our design and of the optimization of such a system is essentially the purpose of the present article.

The effective increase of the laser beam power at the Compton IP can be obtained with two types of optical systems, namely a Fabry-Perot resonator [8] or an optical recirculator [9-11]. Fabry-Perot resonators are indeed best suited for high repetition rate lasers (above tens of megahertz) because the laser oscillator has to be locked to the cavity round-trip frequency with a very high accuracy and therefore with a feedback bandwidth between $10 \mathrm{kHz}-$ $1 \mathrm{MHz}$. Two- and four-mirror cavities have been successfully used to produce $\gamma$-ray beams in the continuous $[12,13]$ and high repetition rate regime $[14,15]$. Another technique, namely the amplifier burst mode [16], is also being investigated but very few results have been reported so far. Cavities containing a frequency converter medium are also considered to equip Compton scattering IPs [17] but they were found to be limited by nonlinear effects [18].

Since the ELI-NP-GBS operates in multibunches train mode at $100 \mathrm{~Hz}$, we choose to design a passive optical system able to recirculate and focus several tens of time a single laser pulse at the Compton IP. In addition, this recirculator must at the same time assure a minimal variation of the $\gamma$-ray central spectral line, by preserving a constant crossing angle $\phi$, between laser and electron beam and optimizing its flux. Its geometry therefore differs from those encountered in laser amplifier [19] and optical delay line $[20,21]$ techniques.

In this article, we give a detailed description of the design, the geometrical optimization, and of the performances of this new kind of optical recirculator. In the next section we describe the conception of a multipass recirculator and the constraints that have to be taken into account. Then, in Sec. III, we apply this design to the case of ELI-NP-GBS and deduce the relevant geometrical parameters. Section IV is devoted to numerical details and to fast simulation tools. The subsequent Sec. V introduces the methods for alignment, synchronization, and monitoring of operation. The final Sec. VI describes the tolerances and expected performances.

\section{OPTICAL RECIRCULATOR FOR COMPTON SCATTERING}

The principle of our planar confocal recirculator is illustrated in Fig. 1: two confocal concave mirrors reflect a circulating Gaussian beam [8] back and forth by forcing it to cross the electron beam axis (i.e., the $z$ axis) at the focus point (i.e., the IP). The sequence of lenses equivalent to a "round-trip" is also shown in Fig. 1. A proper choice of the mirror radii of curvature focusing and collimating sequences alternate.

To provide a quasimonochromatic $\gamma$-ray beam, it is further important to maintain a constant crossing angle $\phi$ at the IP since the central line of its energy spectrum depends on the collision angle [5]. Further, it is imperative to preserve polarization and quality of the wave front.

The geometry coming out from these considerations is the "dragon-shape" recirculator depicted in Fig. 2. The proposed recirculator is made of two big confocal parabolic reflectors for the quality of the wave front after each pass. For a constant angle $\phi$, the laser-electron beams interaction

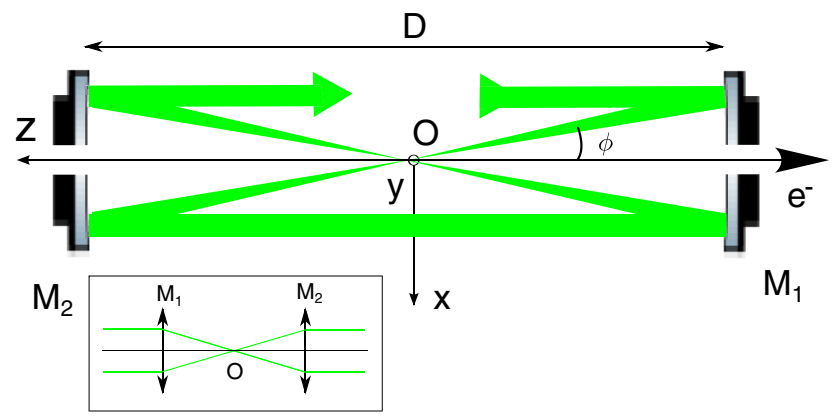

FIG. 1. Illustration of the principle of the planar confocal recirculator and its equivalent sequence of lenses. Note also the coordinate system used throughout this article. 


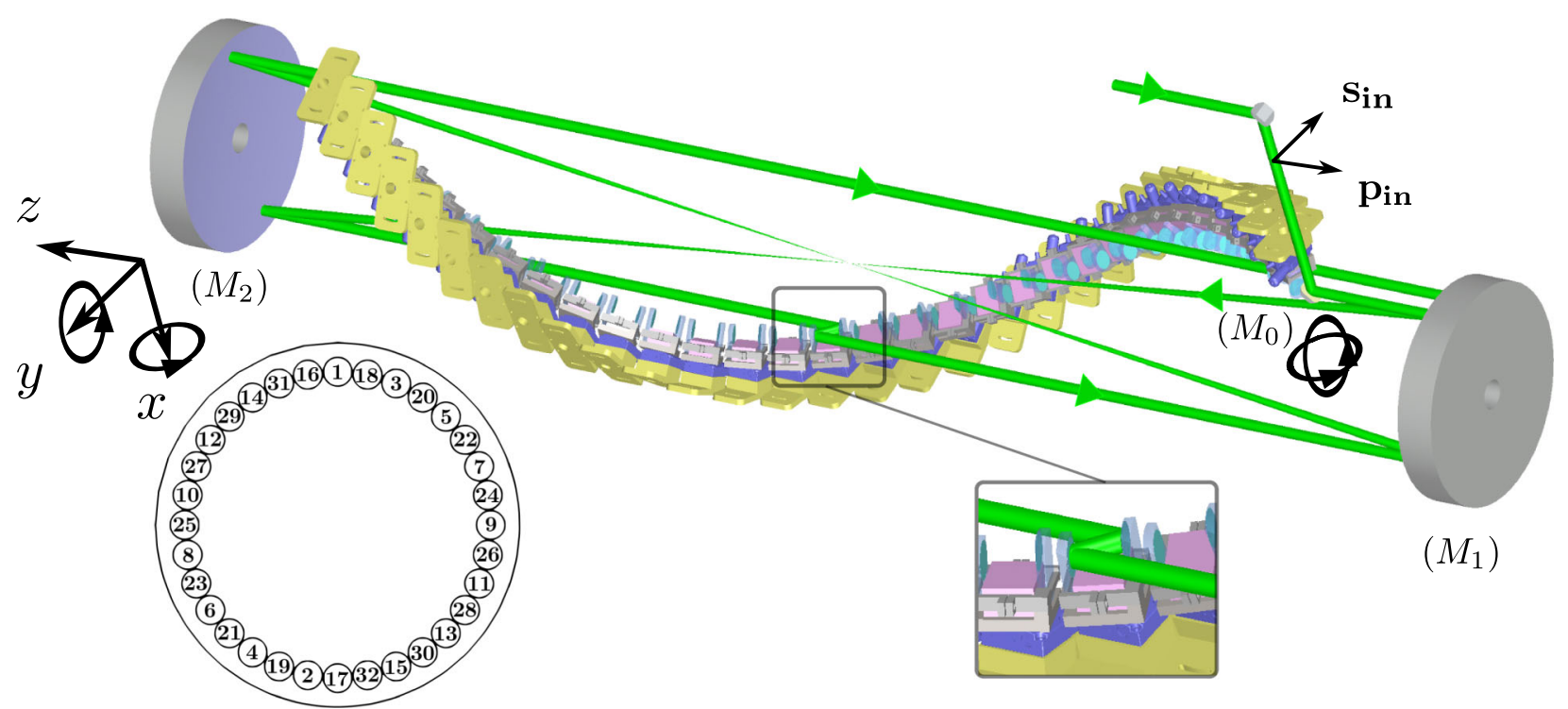

FIG. 2. Isometric view of ELI-NP-GBS recirculator. The mirror $M_{0}$ is used to inject the incident laser beam. The mirror-pair system (structures positioned on a circular helix) and the laser beam paths (green lines) are located between two parabolic mirrors $M_{1}, M_{2}$. Two of the 32 recirculation passes (green lines) are drawn. The polarization vectors $\mathbf{s}_{\text {in }}$ and $\mathbf{p}_{\text {in }}$ related to the incoming laser beam are also shown. The 7 degrees of freedom for the mirror motions are sketched: two tilts for $M_{0}$; two tilts and three translations for $M_{2}$. The inset scheme shows the optical pass ordering.

plane must adapt if more than two recirculations are foreseen. Therefore, two parallel flat mirrors are used to switch from one interaction plane to the next one while keeping the optical axis parallel to the $z$ axis (see Fig. 3). Such a pair of parallel mirrors forms an optical invariant providing an emerging beam parallel to the entrance independently of its orientation. The recirculating laser beam axis is thus always parallel to the symmetry axis $z$ when impinging the parabolic reflectors. This, incidentally, reduces the optical aberrations [22]. In particular, it is well known that when an off axis incident beam is collinear to the parabola axis no astigmatism is induced after reflection [22]. One may note that by construction, as shown in Fig. 2, the mirror-pairs systems (MPS) are located on a circular helix.

The optical system proposed in [9] is also using a sequence of parabolic and flat mirrors leading to the same equivalent lens sequence as shown in Fig. 1. However, the IP positions slips pass after pass along the electron propagation axis whereas in our design the IP is fixed at the same position for all passes.

Since the recirculator has to be installed in the LINAC environment, additional specific constraints must be taken into account.

\section{A. Design constraints: Timing synchronization}

The time structure of the electron bunch train is determined by the radio frequency (rf) accelerating cavities. Thus, the laser pulse frequency for the roundtrip must then be tightly matched to a rf harmonic value.
However, the recirculator round-trip lengths are fixed by the parabolic mirror radii of curvature, i.e., by the confocality condition. This means that the only way to adjust the round-trip length is to make use of the MPS. Its rotation around its own axis $\Gamma$ passing between the two parallel flat mirrors as shown in Fig. 3, changes the

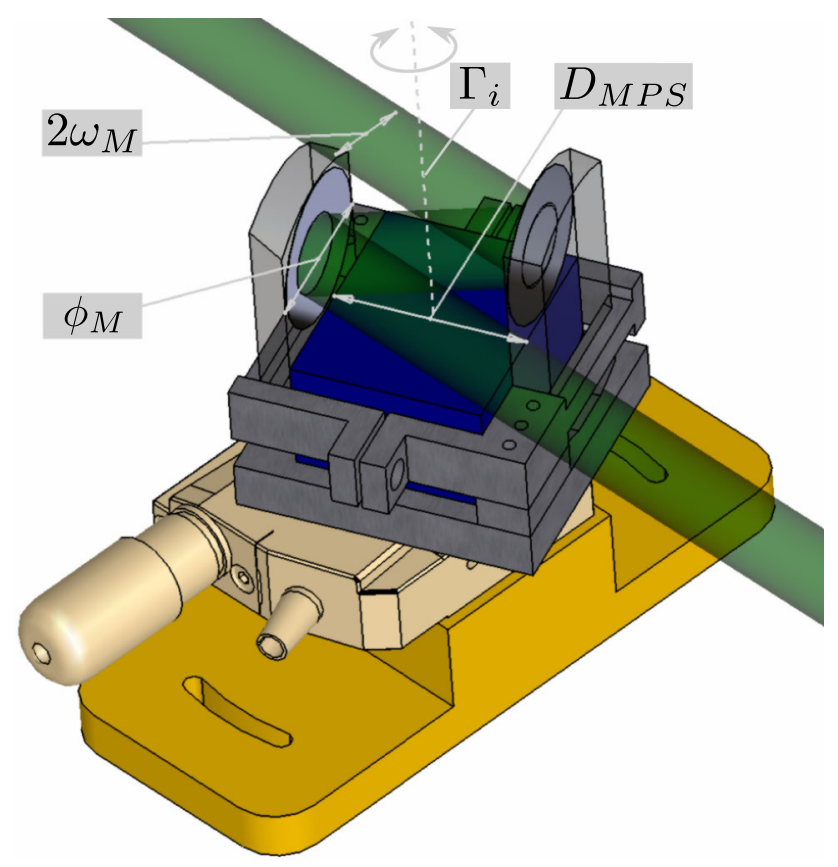

FIG. 3. Isometric view of the $i$ th mirror-pair system and the laser beam path (green line) reflecting on the two parallel mirrors spaced by $D_{\text {MPS }}$. 
incident angle on the mirrors and consequently the optical path length within the MPS. Moreover, this change is made without modifying the laser beam direction as it is independent of the MPS orientation. Therefore a MPS must be implemented before each pass at the IP in order to adjust the timing of each optical recirculation independently. This implies that the interaction plane made by the incident laser pulse and the electron bunch directions is rotated before each bunches crossing. Note that without the requirement for this synchronization only one MPS after two passes would be needed.

The total duration $\tau$ of the rf pulse supplied on the accelerating cavities is limited in time. Hence, the total number of laser pulse recirculations is limited as well by this parameter. The maximal capacities of the photoinjector to generate a large number of electron bunches within the rf pulse duration, which is typically a fraction of a microsecond, has also to be taken into account.

\section{B. Design constraints: Geometrical and optical considerations}

For the design of the recirculator, we have to take into account various parameters of the laser beam and some geometrical constraints that are discussed below.

\section{Laser fluence damage threshold of reflective surfaces}

The laser pulse peak power is a constraint for the beam spot size on the mirrors and therefore on the overall geometry (focal length, laser beam waist, and size of the optics). The laser beam radius $w_{M}$ on a surface is directly linked to its fluence $F$ on this surface. Using $F=2 U / \pi w_{M}^{2}$ and requiring $F \leq F_{\max }$, we obtain

$$
w_{M} \geq \sqrt{\frac{2 U}{\pi F_{\max }}},
$$

where $U$ is the circulating laser pulse energy and $F_{\max }$ the maximum fluence corresponding to the damage threshold for the mirror coating. Using this expression, we also get the distance $D$ between the two vertices of the parabolic mirrors (total length of the recirculator) [8]:

$$
w_{M} \approx \frac{\lambda}{\pi w_{0}} M^{2} \frac{D}{2} \Rightarrow D \geq \frac{2 \pi w_{0}}{\lambda M^{2}} \sqrt{\frac{2 U}{\pi F_{\max }}},
$$

where $w_{0}$ is the beam waist at the IP, and $\lambda$ the central laser wavelength. $M^{2}$ describes the laser beam wave front quality [23]. It is defined as the ratio of the angle beam divergence over the angle beam divergence of the corresponding diffraction-limited Gaussian beam. Since the circulating laser beam is collimated between the two parabolic mirrors, we further set the size of the effective diameter $\Phi_{M}$ of the MPS mirrors (see Fig. 3) by

$$
\Phi_{M}=2 n_{d} w_{M},
$$

where $n_{d}$ is an arbitrary number which controls the diffraction loss (i.e., $\approx \exp \left(-2 n_{d}^{2}\right)$ for a circular aperture).

\section{Mirror surfaces quality}

Diffraction losses can be induced e.g., by local defects (scratches and digs [24] or roughness [25]). However, since a high damage threshold already requires the highest grade mirror surface quality [26], one can safely neglect these losses which currently are well below the $10 \mathrm{ppm}$ level.

Another global defect is the deviation of the mirror surface from its design shape. Such a phenomenon can come from imprecise substrate polishing or from the surface stress exerted by the coating on the substrate. Again, considering a large number of passes, this may induce optical aberrations and variations of the beam waist on the recirculating laser beam. This effect can be assessed by means of a dedicated simulation study as was done in [27]. In the case of our recirculator, we have to match the radii of curvatures of the two parabolic reflectors. Otherwise the laser beam waist will decrease or increase all along the optical passes.

Finally, high reflectivity mirrors are required in order to minimize the transmitted power losses. This imposes the choice of multilayer dielectric coatings.

\section{3. $\gamma$-ray spectral width}

Several factors, including the acceptance angle, beam quality, and laser pulse parameters, are indeed limiting the finesse of the $\gamma$-ray spectral width $[5,6,28,29]$. Accordingly, the full width at half maximum of the laser spectral distribution $\Delta \nu_{L}$ must be limited and, due to detrimental effects of ponderomotive forces and nonlinearities, the laser pulse intensity at the IP must be well below the critical intensity for multiphoton absorption [29]. This threshold is usually characterized by the laser pulse peak normalized amplitude [6]

$$
a_{0}=4.3\left(\frac{\lambda}{w_{0}}\right) \sqrt{\frac{U[\mathrm{~J}]}{\sigma_{t}[\mathrm{ps}]}},
$$

where $\sigma_{t}$ is the root mean square of the laser pulse time distribution. To ensure a small $\gamma$-ray linewidth, one must impose $a_{0} \ll 1$ and therefore some constraints on $U, w_{0}$, and $\sigma_{t}$ for a given $\lambda$, see Sec. III A.

\section{Laser beam polarization transport}

When $\gamma$ rays are used for nuclear physics experiments, particular attention must be paid to the laser beam polarization. Normally, the laser beam should exhibit linear polarization and the recirculator must preserve the polarization state, cf. Sec. VID. Note that for other applications, 
e.g., $\gamma-\gamma$ collider [30] or positron polarized source [31], it has to be circularly polarized.

\section{Azimuth ordering of the optical passes}

It can be easily figured that laser beam spots on the parabolic reflectors describe a circle of radius $R_{c}$ centered on the $z$ axis (see Fig. 4). It can be shown that the position on the parabolic reflector of the laser beam spots regularly jumps to the opposite diagonal describing twice the perimeter of the circle. As shown in Fig. 2, the number of laser spots must then be a multiple of four and consequently located at equal distance $D_{\perp}$ on this circle as all MPS are similar.

\section{Tolerances on optical elements sizes, mechanical stroke, and thickness of mirrors and mounts}

Dimensional tolerances from the manufacturing process of the optical and mechanical components must be taken into account in the final design. The most important parameters are (see Fig. 5) the distance $D_{\text {MPS }}$ between the two mirrors constituting a MPS and the tolerance of its value, the mirror mounts thickness $e$, the distance $D_{M}$ between reflective surfaces of two adjacent MPS ( $e$ added

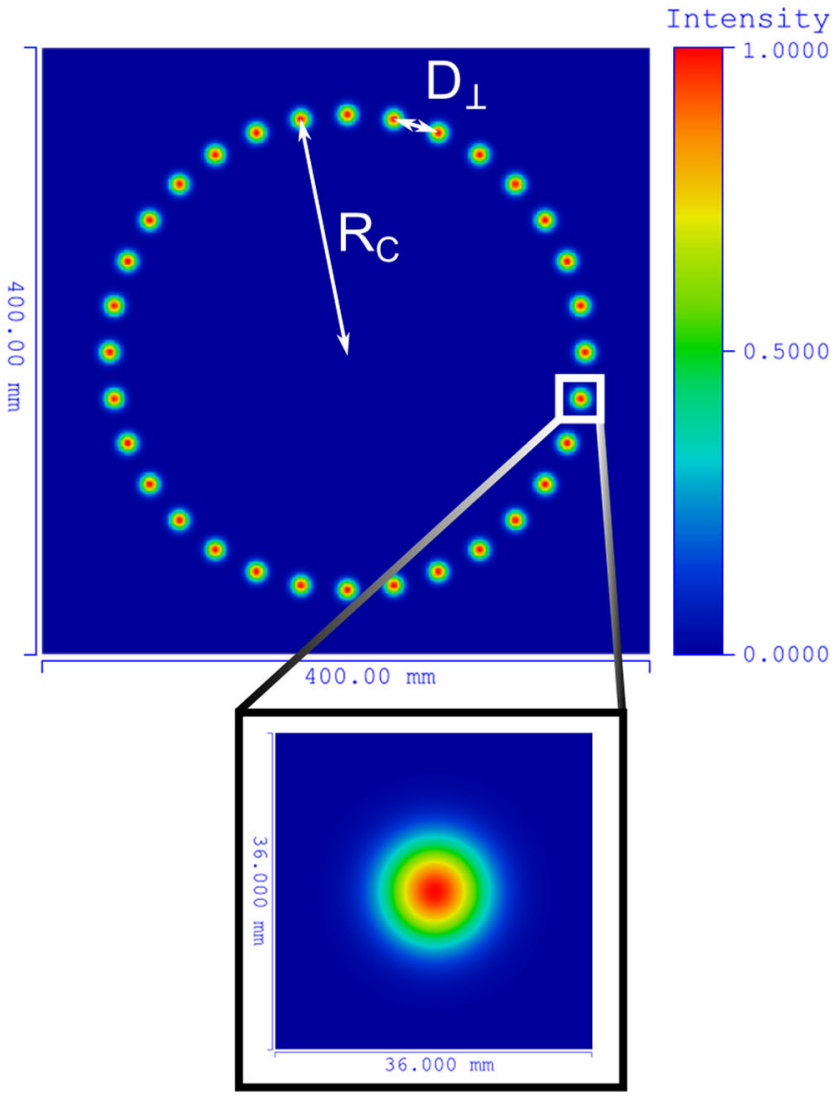

FIG. 4. Beam intensity profiles of the focusing parabolic mirror as computed by the CODE V software. The intensity distribution of the last pass is zoomed and shown separately.

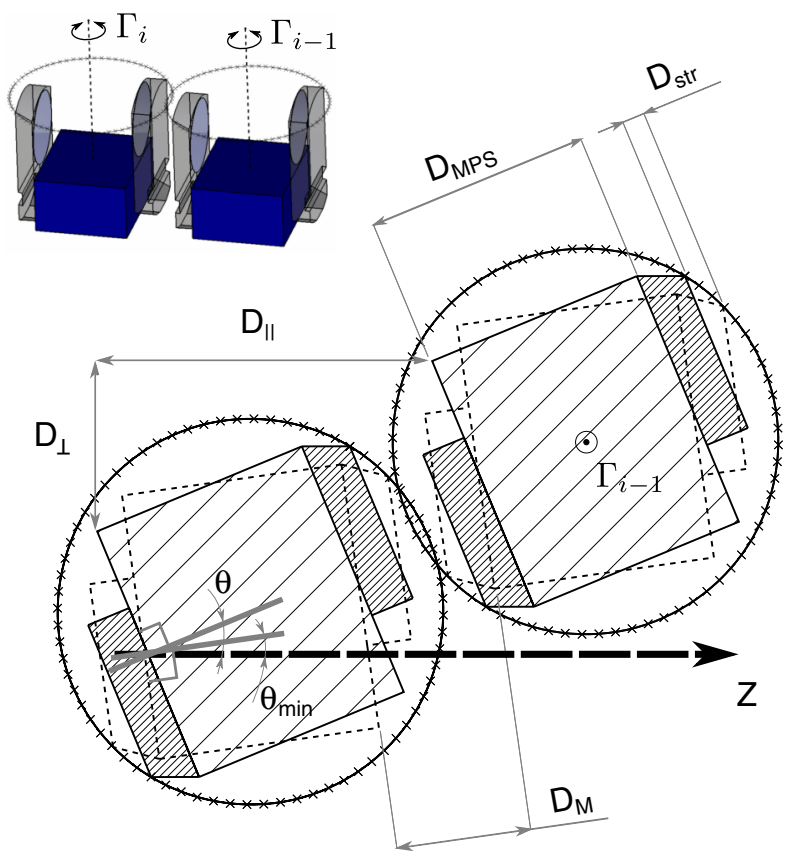

FIG. 5. Two MPS disposition scheme (unrolled view) from the top view of Fig. 3 and the corresponding isometric view given in the inset are shown. The MPS's stroke (circles with crosses), the initial position (hatched) with the corresponding incident angle $\theta$, and the maximal rotated MPS (dotted) with the minimal incident angle $\theta_{\min }$ are represented. $D_{\text {str }}$ is the stroke generated when the MPS is in the maximal rotated position with respect to the initial position. $D_{\perp}$ and $D_{\|}$are the distances between the centers of two neighboring MPS in the $x y$ plane and along the $z$ axis, respectively.

to the mirror thickness and a safety margin), and the tolerance of focal lengths of the parabolic mirrors.

All these parameters are correlated in a complex way (see Appendix A). Parabolic reflector focus points must be tightly aligned on top of each other. However, since the reflectors will have a sizable focal uncertainty as available from the providing firms, all the other distance parameters, and the MPS orientation angle $\theta$, must be tuned to adjust the recirculator round-trip length to a harmonic of the $\mathrm{rf}$ wavelength while maximizing the number of passes and satisfying the previous constraints. This procedure is described in detail in Appendix A.

\section{Maximum number of recirculation passes}

In order to optimize the TASD of the $\gamma$-ray beam, which is one of the most important figures of merit for photonuclear application, we have to maximize the number of passes $N_{\text {pass }}$ while reducing the crossing angle $\phi$. As these two parameters act in an opposite way on the TASD [5] an optimum must be found. There are three main limitations to $N_{\text {pass }}$ : the maximum number of spots spaced by $D_{\perp}$ that one can position on the circle of radius $R_{c}$, the number of passes during a time $\tau$, and the maximum number of MPS spaced 
by $D_{\|}$that one can insert on the circular helix over a distance (pitch) $D$. We thus obtain

$$
N_{\text {pass }} \leq \min \left\{\frac{2 \pi R_{c}}{D_{\perp}}, \frac{c \tau}{2 D}, \frac{D}{D_{\|}}\right\}
$$

A realistic value of $N_{\text {pass }}$ must account for the abovementioned constraints and it also must be a multiple of four. The equations that we use to determine the actual value of $N_{\text {pass }}$ are given in Appendix A.

\section{SYSTEM OPTIMIZATION PROCEDURE}

From the previous section, one can notice that the system is determined by the following set of parameters: $\lambda, U, M^{2}$, $F_{\max }, n_{d}, w_{0}, \phi, \tau$, and $\nu_{r f}$. The latter is the rf applied to the accelerating cavities. Turning now to the optimization of the system one immediately realizes that some of these parameters are strongly dependent on the technology choices for the laser and the accelerator. The following discussion is based on the given parameters for the proposed ELI-NP-GBS facility. The remaining free parameters can be optimized to maximize the TASD of the $\gamma$-ray beam.

\section{A. The parameters of the $\gamma$-ray beam source of ELI-NP-GBS}

For ELI-NP-GBS, the LINAC length and capabilities and the $\gamma$-ray beam characteristics impose strong constraints on the laser beam wavelength $\lambda$, the highest possible laser pulse energy $U$, with a pulse repetition rate of $100 \mathrm{~Hz}$ and a pulse width $\sigma_{t}$ of the order of picoseconds. Taking the state of the art of the high power ytterbium laser technology [32] we here adopt $U=400 \mathrm{~mJ}, \lambda=515 \mathrm{~nm}$, $M^{2}=1.2$, and $\sigma_{t}=1.5 \mathrm{ps}$. These parameters and those of the electron beam are summarized in Table I.

As highlighted in Sec. II B, the reflection coating damage thresholds, $U$ and $\sigma_{t}$ give a strong constraint on the recirculator geometry. We assume that the realistic value $F_{\max }=20 \mathrm{~J} / \mathrm{cm}^{2}$ for $\sigma_{t}=4.2 \mathrm{~ns}$ that we extrapolate to the picosecond regime using the empirical law derived in Ref. [33]. This leads to $F_{\max }=0.38 \mathrm{~J} / \mathrm{cm}^{2}$ for $\sigma_{t}=1.5 \mathrm{ps,}$ compatible with today's best commercially available mirror coatings.

For ELI-NP-GBS, the pulse generation from the photoinjector of the electron accelerator method and the characteristics of its accelerating rf cavities (beam loading effect, beam breakup, and $\tau=600 \mathrm{~ns}$ ) constrain $N_{\text {pass }} \leq$ 32 for a minimum bunch spacing of $15 \mathrm{~ns}$. In addition this leads to bound the overall recirculator length (distance between the two parabolic mirrors) $2.3 \mathrm{~m}<D<2.8 \mathrm{~m}$.

The parameter $n_{d}$ is chosen in order to minimize the diffraction losses induced by the vignetting on the MPS. It is fixed to $n_{d}=1.8$.
TABLE I. Summary table of the adopted electron beam and laser beam parameters.

\begin{tabular}{lc}
\hline \hline Parameters & For $10 \mathrm{MeV} \gamma$ ray \\
\hline Laser beam & \\
$\mathrm{U}(\mathrm{mJ})$ & 400 \\
$\sigma_{t}(\mathrm{ps})$ & 1.5 \\
$M^{2}$ & 1.2 \\
$\lambda(\mathrm{nm})$ & 515 \\
Pulse repetition rate $(\mathrm{Hz})$ & 100 \\
Electron beam & \\
Energy $(\mathrm{MeV})$ & 520 \\
Bunch charge $(\mathrm{pC})$ & 250 \\
Normalized rms emittance $(\mathrm{mm}$ mrad) & 0.4 \\
Bunch length rms $(\mu \mathrm{m})$ & 280 \\
Focal spot size rms $(\mu \mathrm{m})$ & 20 \\
$\nu_{r f}(\mathrm{GHz})$ & 2.856 \\
$\tau(\mathrm{ns})$ & 600 \\
\hline \hline
\end{tabular}

The last parameter to be fixed is the incident angle of the laser beam on the MPS. As the mirror coatings are made of multidielectric quarter wave stacks [34], we choose $\theta \approx 22.5^{\circ}$ to reduce the change in laser beam polarization after a reflections while keeping enough flexibility to adjust the total length for the round-trip. As shown in Appendix B, a small value of $\theta$ also helps to reduce the parallelism misalignment of the MPS.

In the end, we are left with two free parameters, $w_{0}$ and $\phi$. Which must be determined to maximize the TASD. The beam waist is bounded by $w_{0} \geq 25 \mu \mathrm{m}$ according to the constraints described in Sec. II B 3.

\section{B. TASD calculation}

The Compton cross section is well known [35]. To compute the $\gamma$-ray spectrum, one needs to take the electrons and laser beam spatiotemporal shape into account $[1,3-5,36]$. For a given set of laser beam parameters, we can choose the electron beam beta function at the IP in order to optimize the $\gamma$-ray flux. In our case, when optimizing the recirculator geometry we can also subsequently optimize the electron beam parameters for each possible value of $w_{0}$ and $\phi$.

In the next section, we concentrate on the optimization of the TASD as defined in [5] and we use the expressions of $[1,3,4]$ for numerical calculations. Accordingly, the TASD values for one pass obtained with the beam parameters of Table I are shown in Fig. 6.

\section{Optimization results}

The TASD is computed as a function of $w_{0}$ and $\phi$, using the parameters fixed previously and those calculated with the equations in Appendix A.

The upper plot of Fig. 7 shows the maximum number of passes as a function of $\phi$ and $w_{0}$. In view of the behavior we 


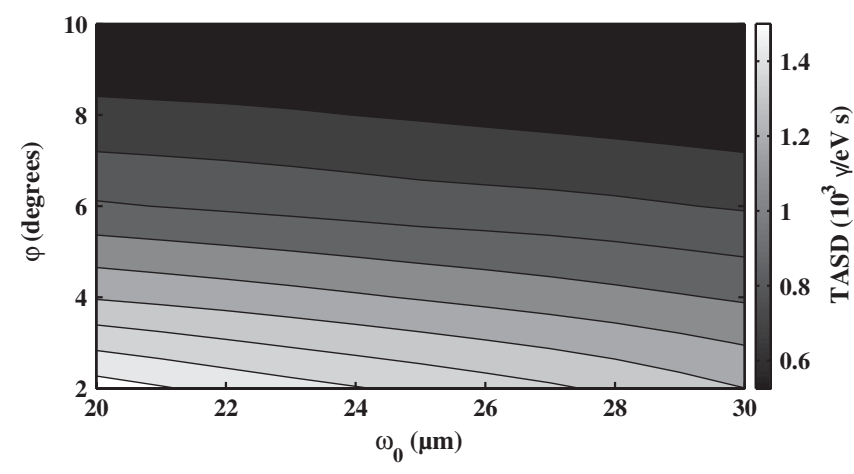

FIG. 6. TASD results for one recirculation pass. The region of interest is the light gray region.

choose $w_{0}=28.3 \mu \mathrm{m}$ as an optimized value (i.e., the value which maximizes the number of passes while reducing $w_{0}$ ).

The TASD corresponding to $w_{0}=28.3 \mu \mathrm{m}$ is shown in Fig. 7(b). The step shape of $N_{\text {pass }}$ for a constant $w_{0}$ comes from its divisibility by 4 while the sawtooth shape of the TASD exhibits the $\phi$ dependence of the cross section combined with the $N_{\text {pass }}$ behavior. From this plot we deduce that the maximum TASD $\left(\sim 2.2 \times 10^{4} \gamma / \mathrm{eV} \mathrm{s}\right)$ is found for $\phi=7.5^{\circ}$ and $N_{\text {pass }}=32$. This choice corresponds to a good compromise between a high number of

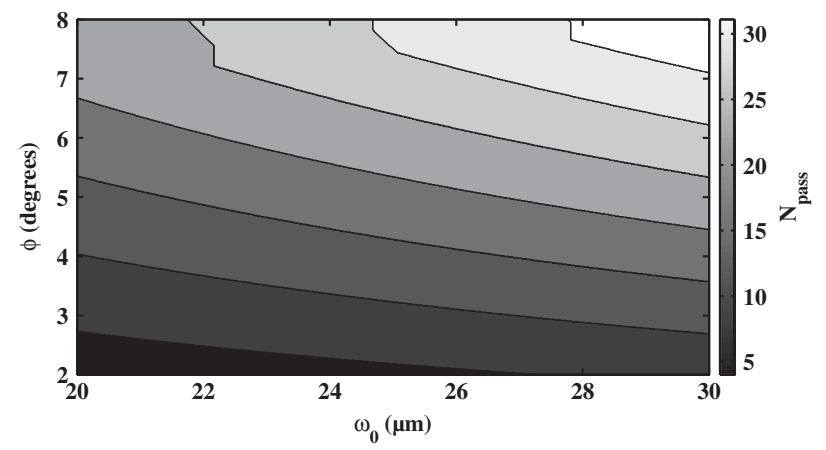

(a)

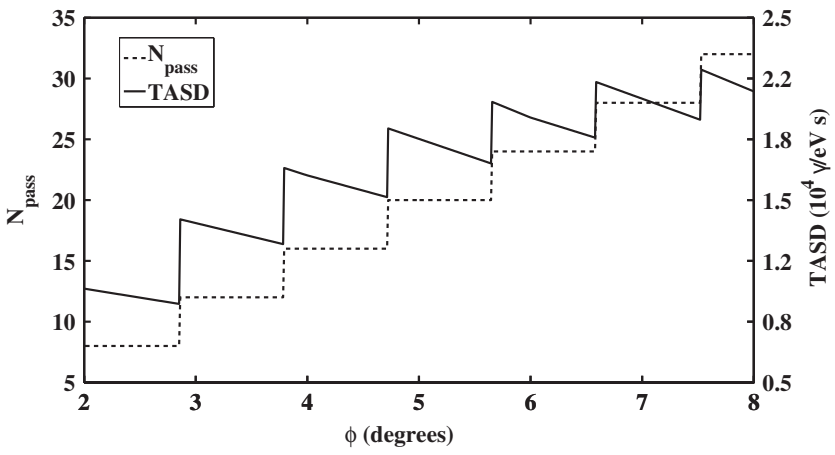

(b)

FIG. 7. (a) The maximum number of passes as a function of $\phi$ and $w_{0}$. The region of interest is the light gray region. (b) The TASD and maximum number of passes as a function of $\phi$ for $w_{0}=28.3 \mu \mathrm{m}$. The results correspond to the $10 \mathrm{MeV} \gamma$-ray beam (see Table I).
TABLE II. Summary table of the recirculator's parameters obtained after optimization with the specified input parameters for ELI-NP-GBS's specifications (see Appendix A for calculations).

\begin{tabular}{|c|c|}
\hline Parameters & For $10 \mathrm{MeV} \gamma$ ray \\
\hline \multicolumn{2}{|c|}{ Input parameters (see Appendix A) } \\
\hline$\Delta \ell(\mu \mathrm{m})$ & 160 \\
\hline$\delta D_{\mathrm{MPS}}(\mu \mathrm{m})$ & 100 \\
\hline$D_{M}(\mathrm{~mm})$ & 27 \\
\hline$e(\mathrm{~mm})$ & 5 \\
\hline$n_{d}$ & 1.8 \\
\hline$w_{0}(\mu \mathrm{m})$ & 28.3 \\
\hline$\phi\left(^{\circ}\right)$ & 7.54 \\
\hline$\theta^{(0)}\left({ }^{\circ}\right)$ & 22.5 \\
\hline $\begin{array}{l}F_{\max }\left(\mathrm{J} / \mathrm{cm}^{2}\right) \text { for } \sigma_{t}=4.2 \mathrm{~ns} \\
\text { Mirror pairs (see Fig. } 5 \text { ) }\end{array}$ & 20 \\
\hline$D_{\mathrm{MPS}}(\mathrm{mm})$ & 40.1 \\
\hline$\theta\left({ }^{\circ}\right)$ & 22.54 \\
\hline$D_{\|}(\mathrm{mm})$ & 60.5 \\
\hline$D_{\perp}(\mathrm{mm})$ & 30.7 \\
\hline \multicolumn{2}{|l|}{ Recirculator (see Figs. 3, 4) } \\
\hline$N_{\text {pass }}$ & 32 \\
\hline$D(\mathrm{~mm})$ & 2378.9 \\
\hline$R_{C}(\mathrm{~mm})$ & 156.7 \\
\hline$w_{M}(\mathrm{~mm})$ & 8.3 \\
\hline$\Phi_{M}(\mathrm{~mm})$ & 29.7 \\
\hline $\operatorname{TASD}[\gamma /(\mathrm{s} \mathrm{eV})]$ & 22000 \\
\hline
\end{tabular}

passes, reasonable crossing angle $\phi$ and low $w_{0}$ which imply a high TASD. Our final parameters are summarized in Table II. These were implemented in a computer-aided design (CAD) software to check the geometrical and practical consistency of the solution.

\section{OPTICAL SIMULATIONS}

Once the recirculator geometry has been optimized, the space coordinates and orientation of all optical elements are known. This information is then used to fully model the recirculator with the CODE V software [37] (128 reflective surfaces were implemented to describe the recirculator geometry of Table II). At this stage, the recirculator is "by definition" perfectly aligned. As a result, using the accurate beamlet basis propagation of CODE V, we did not observe any noticeable optical aberrations nor any significant vignetting losses, even after the 32 passes. The optical quality of the system is illustrated in Fig. 4 where the beam intensity on one of the parabolic reflectors is shown. The nonsequential mode of CODE V (mode which considers all the surfaces at the same time during simulation) was used to produce this figure which demonstrates that the beam quality is preserved until the last pass.

However, tolerance studies for the alignment of the optical components must also be carried out. Assuming that one of the two parabolic reflectors $\left(M_{1}\right)$ is taken as a geometrical reference, one needs to consider $5^{\circ}$ of motion 
on $M_{2}$ mirror (i.e., three translations and two rotations, see Fig. 2) to reach the confocal geometry. Besides, one also needs to move the mirror $M_{0}$ to align the incident laser beam on the recirculator axis $z$. As for the MPS, their parallelism constraint is found to be so strong that it has to be fixed during the manufacturing phase. Consequently, we had to design a dedicated MPS alignment process described below.

Eventually, the tolerable level of misalignment is driven by the induced TASD losses. To determine this acceptable level, one must first choose sequences of tilts and displacements of optical elements (see Appendix B for the MPS parallelism). Then, the 32 optical passes are simulated and the TASD is computed separately for each pass. For a misaligned laser beam, we must then numerically compute the geometrical factor of the TASD, more precisely the luminosity geometrical factor, which consists of a quadruple integral describing the space-time overlap between electron bunches and laser pulses [38] (Table I provides the beams parameters). Thus, the CODE V optical simulations must be complemented by another software, e.g., MATLAB, to perform the geometrical luminosity calculations.

However, with 128 reflective surfaces to simulate, such a procedure turns out to consume an unrealistic amount of computing time. One way to circumvent this problem is to use a fast simulation based on an estimator correlated to TASD losses for a given optical misaligned configuration. We are going to describe this estimator and to show its degree of correlation with the TASD on numerical examples. Prior to this we however need to describe the MPS parallelism alignment procedure and the synchronization tuning.

\section{A. MPS parallelism adjustment}

As mentioned earlier, the tolerance on the MPS parallelism is very tight. Consequently, particular attention has to be paid to this issue. Owing to our simulation, we realized that this is due to the large number of passes. We then choose to follow the procedure depicted in Fig. 8 to reduce the cumulative misalignment that appears when adjusting the MPS parallelism independently of the others. Accordingly, the $n$th MPS parallelism $\varepsilon_{n}$ is adjusted by taking into account the residual cumulative misalignment of all the $n-1$ previous MPS (i.e., $\sum_{i=1}^{n-1} \varepsilon_{i}^{\prime}$ ). In this way, the parallelism precision $\Delta \varepsilon^{\prime}$ is independent on the number of MPS as long as the MPS sequence is kept inside the recirculator. Precision of the order of a few microradians can be reached with modern numerical glass polishing techniques.

A model of the alignment procedure is given in Appendix B. It provides the correspondence between the sequence of 32 MPS default parallelism and the apparatus precision used in the manufacturing process. That is the relationship between $\varepsilon$ (the angle between the two flat mirrors of one MPS) and $\varepsilon^{\prime}$ (or $\varepsilon^{\prime \prime}$ if an autocollimation

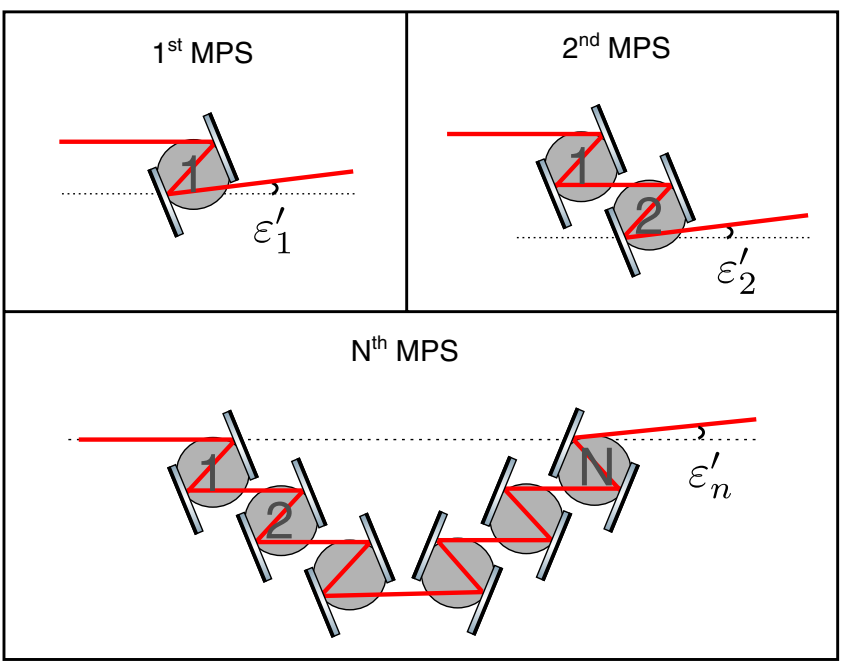

FIG. 8. Illustration of the MPS alignment for parallelism. The equations that describe this method are presented in Appendix B.

method is used, see Fig. 22). For example, loose precision values for the autocollimator $\Delta \varepsilon^{\prime \prime}$ result in the distributions of $\varepsilon$ shown in Fig. 23.

In the following, we shall define $\Delta \varepsilon$ as the average value of the $\varepsilon$ distributions of the MPS (see Appendix B). Series of misaligned MPS were implemented in our CODE V simulations. We used the recirculator and beam parameters of Tables II and I assuming that the parabolic reflectors and injection are properly aligned (the effect of the parabolic mirrors and injection alignment is described in Sec. VI B). Figure 9 shows the statistical distribution of the TASD losses as a function of different values of $\Delta \varepsilon$ when the procedure of Fig. 8 is used and when MPS parallelism are adjusted independently of one another. Although a reflection occurs on each parabolic reflector between two successive MPS, the procedure of Fig. 8 leads to an

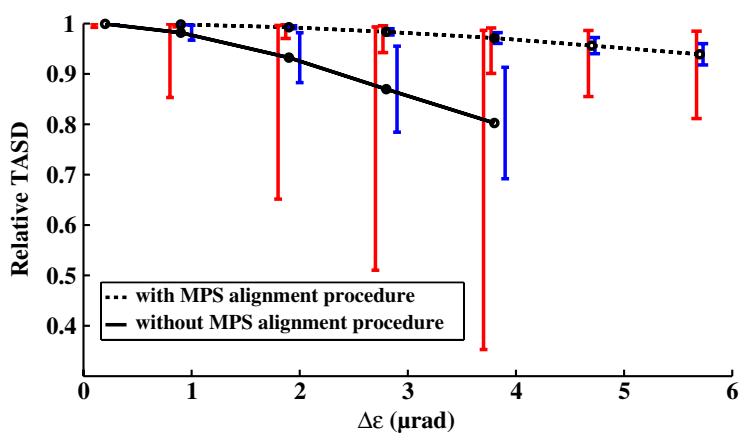

FIG. 9. Statistical distribution of the relative TASD as a function of MPS parallelism (see Appendix B) with 5000 events per point. The configurations are simulated with the fast MATLAB code [39] described in Sec. IV C. The points connected by a dashed (solid) line describe the average relative TASD of the data with (without) the MPS alignment procedure. The error bars on the left of each point represent the extreme events of the data and the error bars on the right of each point represent the rms. 


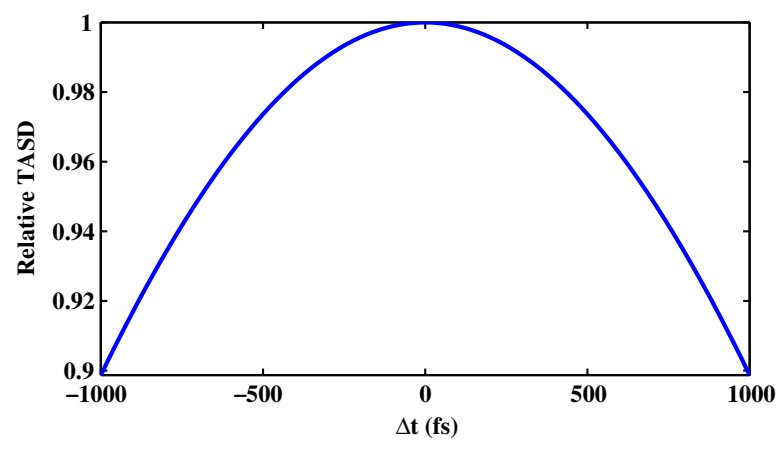

FIG. 10. Relative TASD as a function of synchronization delay between laser and electron pulses. Note that the function is Gaussian.

important decrease of the impact of MPS parallelism misalignment on TASD.

\section{B. Synchronization tuning (MPS rotation)}

The synchronization between circulating laser pulses and electron bunches must be tuned independently for the 32 optical passes. As indicated in Sec. II, this is done by rotating around $\Gamma_{i}$ the MPS independently of each others (see Fig. 3). Taking the parameters of Table II, we calculated that a MPS must be rotated by $20 \mathrm{mrad}$ to compensate for a 2 ps synchronization delay. This rotation generates a transverse shift variation of the outgoing laser beam of $1.5 \mathrm{~mm}$. As shown in Appendix B, this shift is, in first approximation, independent of the residual MPS parallelism misalignment. We checked that, using the nonsequential CODE V simulation mode, vignetting losses induced by this shift are very small and lead to a negligible effect on TASD.

Figure 10 shows relative TASD (TASD/TASD ${ }_{\max }$ ) as a function of the delay between the laser pulse and the electron bunch. To ensure optimal overlap between the electron bunch and the laser pulse, and to optimize the TASD, a synchronization accuracy of about $\pm 200 \mathrm{fs}$ is required to keep the induced losses below the percent level.

We identified two ways to measure the synchronization delay, the first uses the $\gamma$-ray flux measurement directly and the second a dedicated interferometric calibration that will be described in Sec. V.

\section{A geometrical estimator for TASD: Shortest distance between beam axes (SDBA)}

At this stage it is possible to formulate an estimator for the TASD losses of misaligned recirculators. First, one may note that the Rayleigh length [8] $z_{R}=\pi w_{0}^{2} /\left(M^{2} \lambda\right)$ of the recirculating laser beam (here we have $z_{R}=4.1 \mathrm{~mm}$ ) is more than 1 order of magnitude higher than the lengths of the electron bunch and the laser pulse (see Table I). Second, in the case of only small optical misalignments, the use of parabolic reflectors preserves the laser beam waist and keeps the optical aberrations at a negligible level. The transverse profile of the laser intensity is thus quasiconstant over the interaction region. Therefore, the shortest distance between the electrons and laser propagation axes (SDBA) could serve as TASD estimator to speed up the simulation. More precisely, for a given recirculator misalignment, the optimum space-time overlap of the electrons and laser beam will occur when the laser pulse will be synchronized to the electron bunch at the SDBA.

To demonstrate the correlation between the TASD and the SDBA, we again use the recirculator parameters of Table II, taking $\Delta \varepsilon=3.8 \mu \mathrm{rad}$ for the sequences of misaligned MPS and choosing the injection and parabolic mirrors perfectly aligned. An ensemble of misaligned MPS configurations is then simulated using CODE V and TASD are finally computed using the MATLAB software [39]. Figure 11(a) shows TASD as a function of the SDBA. This plot contains all optical recirculations of 400 randomly misaligned recirculator simulations (i.e., there are 32 entries for each recirculator configuration). A Gaussian fit is also shown on Fig. 11(a) which perfectly matches the data points.

Notice that in all following figures the laser pulses are synchronized with the electron bunches at the SDBA position. As indicated in Sec. II, this is done by rotating the MPS (by at most $12 \mathrm{mrad}$ for the data of Fig. 11). Accuracy of the correlation between the relative TASD and

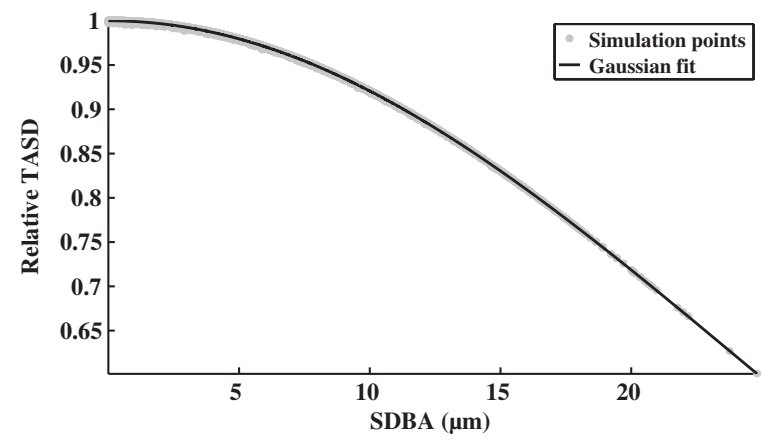

(a)
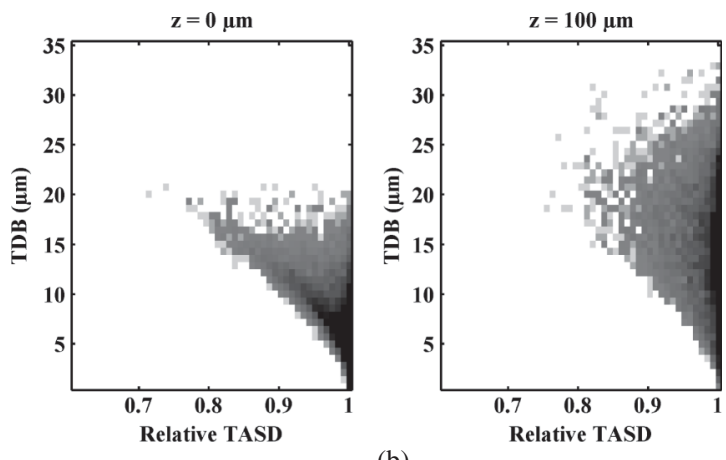

(b)

FIG. 11. (a) The relative TASD as a function of SDBA, note the perfect fit to the data points. (b) The relative TASD as a function of TDB for different transverse positions of the reference plane: left: $z=0 \mu \mathrm{m}$, right: $z=100 \mu \mathrm{m}$. 
SDBA is nearly perfect as the average difference between the fit and the data is equal to $-3 \times 10^{-4}$ and the standard deviation is equal to $5 \times 10^{-4}$. Thus, we are allowed to circumvent the CODE V simulation by using a simple ray tracing algorithm for the laser beam propagation and the Gaussian function to derive the corresponding TASD. Finally, our simulations of the recirculator misalignments are then done solely with a simple and thus fast MATLAB code.

For the sake of simplicity we label our random simulation set as

$$
\xi_{N}(\Delta \varepsilon, \Delta \Theta, \Delta \Xi),
$$

where $N$ is the number of configurations simulated, $\Delta \varepsilon$ is the tolerance on the MPS parallelism as described in Appendix $\mathrm{B}, \Delta \Theta$ and $\Delta \Xi$ are the tolerances on the 7 degrees of freedom, respectively for the four rotations (two for injection and two for one parabolic mirror) and for the three translations (for the parabolic mirror) as represented in Fig. 2. It means that each degree of freedom is randomly chosen in the range $[-\Delta \Theta, \Delta \Theta]$ and $[-\Delta \Xi, \Delta \Xi])$ for the rotations and for the translations, respectively.

\section{ALIGNMENT AND SYNCHRONIZATION TOOLS FOR ELI-NP-GBS}

\section{A. An optical estimator for the alignment: The transverse distance to barycenter (TDB)}

Although it would be in principle possible to measure directly SDBA. It however appears unrealistic to implement a detection system inside the recirculator. Instead, we need to build a robust optical estimator capable to provide a guideline for the optical alignment of the recirculator. The simplest solution is the intersection point $\left\{x_{n}, y_{n}\right\}$ of the $n$th optical pass with the plane $z=0$ (i.e., the IP). Measurements of $\left\{x_{n}, y_{n}\right\}$ can be performed inserting a thin pellicle at the recirculator center and using an ultrafast CCD. The principle scheme is given in Fig. 13. In fact, since the exact position of the electron beam axis cannot be known a priori with respect to $\left\{x_{n}, y_{n}\right\}$, we define, for the $n$th recirculation pass, the distance between its intersection point and the barycenter of all 32 intersection points:

$$
\mathrm{TDB}_{n}=\left[\left(x_{n}-\frac{1}{32} \sum_{i=1}^{32} x_{i}\right)^{2}+\left(y_{n}-\frac{1}{32} \sum_{i=1}^{32} y_{i}\right)^{2}\right]^{1 / 2} \text {. }
$$

For the configurations $\xi_{400}(3.8 \mu \mathrm{rad}, 0,0)$ [cf. Eq. (3)] of Sec. IV C, Fig. 11(b) shows the correlations between TASD and TDB measured in two planes $z=0 \mu \mathrm{m}$ and $z=100 \mu \mathrm{m}$. From this figure, one can conclude that the longitudinal position along the $z$ axis does not act much upon the minimal relative TASD for a given TDB. From an experimental viewpoint, this fact will facilitate the pellicle insertion at the recirculator center.
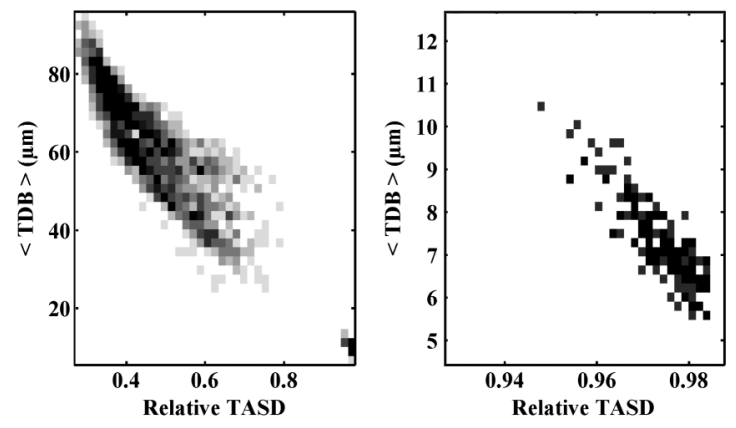

FIG. 12. Relative TASD as a function of $\langle\mathrm{TDB}\rangle$ at the plane $z=0 \mu \mathrm{m}$. Left: $\xi_{400}(3.8 \mu \mathrm{rad}, 0,0)$ (small area in the bottom right corner) mixed with $\xi_{3200}(3.8 \mu \mathrm{rad}, 20 \mu \mathrm{rad}, 20 \mu \mathrm{m})$ (large area in the upper left part of the plot). Right: $\xi_{400}(3.8 \mu \mathrm{rad}, 0,0)$, i.e., zoom on the small area in the bottom right corner of the left plot.

In Fig. 12, we show the average TDB defined by $\langle\mathrm{TDB}\rangle=\sum_{n=1}^{32} \mathrm{TDB}_{n} / 32$ as a function of the TASD loss for the set of misaligned recirculator configurations of Sec. IV C [ $\left.\xi_{400}(3.8 \mu \mathrm{rad}, 0,0)\right]$ with $\xi_{3200}(3.8 \mu \mathrm{rad}$, $20 \mu \mathrm{rad}, 20 \mu \mathrm{m}$ ) [cf. Eq. (3)]. One sees that requiring a minimal value for $\langle$ TDB $\rangle$ will ensure a minimal level of TASD losses. Note that the barycenter defines the expected electron beam axis position.

The insertion in a perfectly align recirculator of a $2 \mu \mathrm{m}$ thick pellicle (index of refraction $\approx 1.51$ ) at an angle of $45^{\circ}$ with respect to the $z$ axis was modeled using CODE V. The results do not exhibit sizable misalignment $(\langle\mathrm{TDB}\rangle \approx 0.7 \mu \mathrm{m})$.

\section{B. Synchronization of the recirculator round-trips}

At ELI-NP-GBS, the alignment system will also be used for the synchronization: we image the laser beam spot on the same thin pellicle as required for the TDB measurement and we add a laser pulse cadenced on the electron bunch frequency as replicate of the electron beam. By interfering the two laser pulses generate an interference pattern which provides information on the delay between the two pulses. From previous simulation the insertion of the pellicle induces a cumulative time delay below 90 fs. Figure 13 shows the schematic principle of the alignment and synchronization tools. This method has the great advantage that we use only a single pellicle for alignment and synchronization. Indeed, there is a significant saving in terms of space occupancy and cost.

Once "optically synchronized," the electron bunches themselves are used for the final tuning. From Fig. 10 one sees that the TASD and consequently the $\gamma$-ray flux is directly linked to the delay between the two beams. Another advantage is that this method might be done on-line and does not require to stop the accelerator and a folded mirror insertion at the IP. For ELI-NP-GBS this method will thus provide the on-line monitoring of 


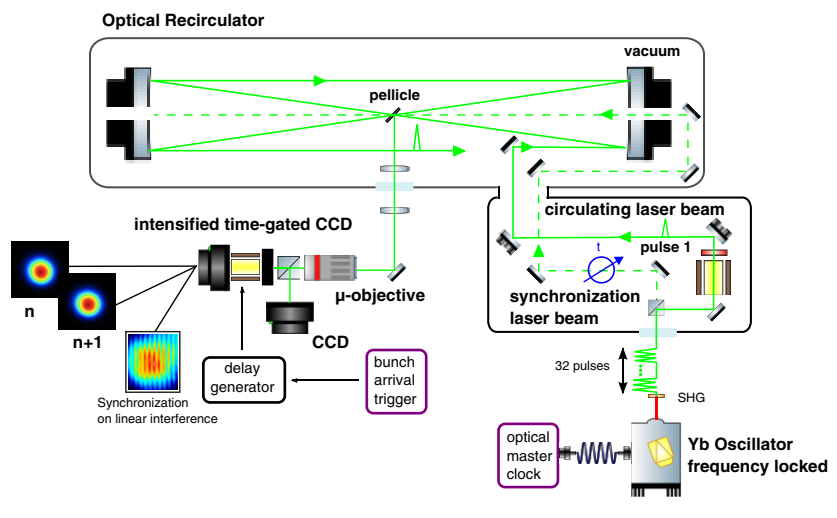

FIG. 13. Principle scheme of the synchronization and alignment tool. A recirculating laser beam (solid line) is injected inside the recirculator from the injection chamber. A thin beam-splitter plate labeled "pellicle" images the spot of each pass at the IP. Finally, the laser spot image is recorded pass after pass by one dedicated imaging system composed of a micro-objective, an intensified time gated CCD and control system (CCD, trigger, delay generator). For synchronization purposes, the synchronization laser (dashed line) is also injected from the injection chamber onto the electron beam axis. Consequently interference pattern is imaged on the pellicle.

the recirculator performance for each optical pass independently.

\section{TOLERANCES AND PERFORMANCES AWAITED FOR ELI-NP-GBS}

\section{A. MPS parallelism tolerance}

We first estimate the tolerances on MPS parallelism misalignment. Using the fast simulation depicted in Sec. IV C, we determined the TASD losses for $\xi_{5000}(\Delta \varepsilon, 0,0)$ with $\Delta \varepsilon=[0.9,1.9,2.8,3.8,4.7,5.7] \mu \mathrm{rad}$ [cf. Eq. (3)]. Figure 9 shows the statistical estimators of the

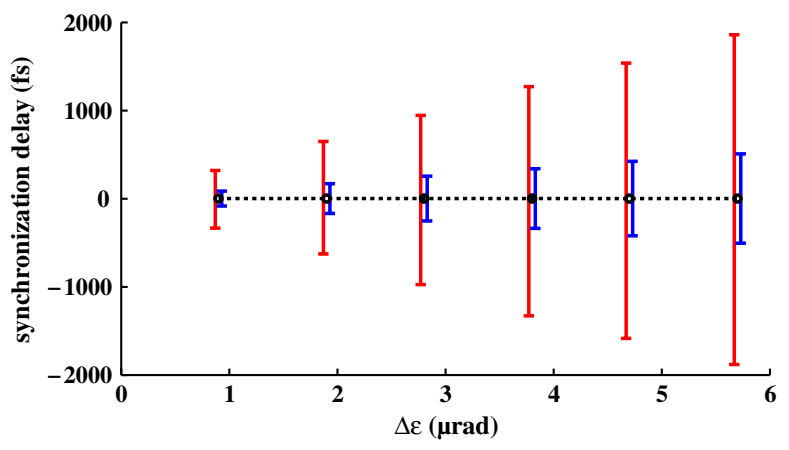

FIG. 14. Statistical distribution of the synchronization delay induced by MPS misalignment with 5000 events per point. The points connected by a dashed line describe the average synchronization delay of the data with the MPS alignment procedure of Appendix B. The error bars on the left of each point represent the extreme events of the data and the error bars on the right of each point represent the rms.
TASD distribution computed from the simulated events. The main statistical estimators related to the distributions of the corresponding synchronization delay are also shown in Fig. 14. From these plots, one sees that in order to keep TASD losses below $10 \%$ with synchronization delay below $1.5 \mathrm{ps}$, one must set $\Delta \varepsilon \leq 3.8 \mu \mathrm{rad}$.

\section{B. Parabolic reflector and laser injection tolerance}

We simulate the TASD losses induced by $\Delta \Theta$ and $\Delta \Xi$ with perfectly aligned MPS (i.e., $\Delta \varepsilon=0$ ). Figure 15 shows the statistical estimators (as in Fig. 9) of the TASD distributions for $\xi_{3000}(0, \Delta \Theta, \Delta \Xi)$ with $\Delta \Theta(\Delta \Xi)=$ $[3,10,20] \mu \operatorname{rad}(\mu \mathrm{m})$ [cf. Eq. (3)]. From this figure, one sees that, to keep the losses below $10 \%$, we have to set the tolerances of these parameters at the level of few micrometers and microradians. Since such tight tolerances cannot be reached with the classical techniques for optical alignment $[40,41]$ the dedicated alignment procedure described in Sec. VIC had to be developed.

\section{Optical alignment of the recirculator}

In Sec. VA we described a simple and robust measurable estimator for TASD losses induced by optical misalignments, the TDB. The aim of the optical alignment procedure is to minimize $\langle\mathrm{TDB}\rangle$ to guarantee high TASD. This is done in two steps. First, the two parabolic reflectors are aligned in front of each other using specific techniques $[40,41]$. This first step leads to residual tilts and displacements of the order of few dozens of microradians and micrometers.

The alignment is refined in a second step using a SIMPLEX minimization algorithm [42]. The $\langle\mathrm{TDB}\rangle$ is thus minimized by acting on the 5 degrees of freedom of one parabola and the two tilts of the laser injection beam (see Fig. 2).

A series of misaligned recirculator configurations $\xi_{2500}(3.8 \mu \mathrm{rad}, 20 \mu \mathrm{rad}, 20 \mu \mathrm{m})$ [cf. Eq. (3)] was simulated. To test the SIMPLEX minimization procedure in a realistic

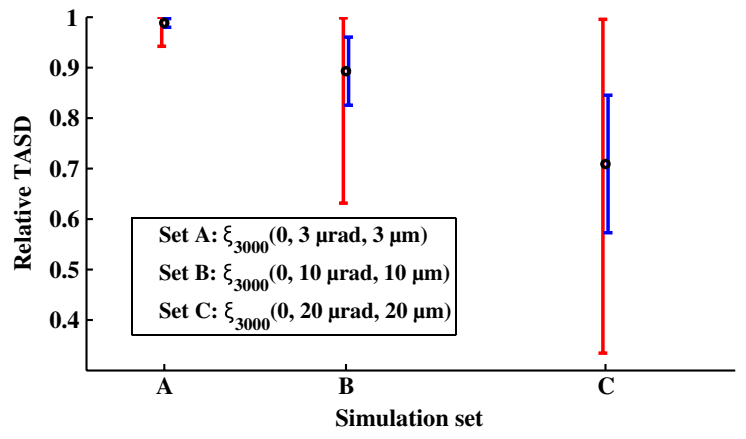

FIG. 15. Statistical distribution of the relative TASD for different values of $\Delta \Theta$ and $\Delta \Xi$ (3000 events per point). The points describe the average relative TASD of the data. The error bars on the left of each point represent the extreme values of the data and the ones on the right of each point represent the rms. 
way we discretized the 7 parameter space. Assuming $1 \mu \mathrm{m}$ and $1 \mu \mathrm{rad}$ for the discretization steps, which correspond to commercially available high quality linear stages and hexapods, we obtain the results of Fig. 16. This figure demonstrates the capacity of the method to optimize the TASD. Starting from $\Delta \Theta=20 \mu \mathrm{m}$, and $\Delta \Xi=20 \mu \mathrm{rad}$ [cf. Eq. (3)] misaligned recirculator with a relative TASD equal to $73 \%$ in average and a rms of $15 \%$, we end up with relative TASD $>95 \%$ after the algorithm. We are thus finally able to reach a TASD values equivalent to a few micrometer, microradian of misalignments. Note that we obtained satisfactory results as long as the provided discretization steps were kept below $3 \mu \mathrm{m}$ and $3 \mu \mathrm{rad}$.

To exhibit the robustness of the algorithm we have run it on a widely misaligned set of configurations $\xi_{1200}(3.8 \mu \mathrm{rad}, 100 \mu \mathrm{rad}, 100 \mu \mathrm{m})$. The results are presented in Fig. 17 and show the high potential of our alignment method. Beginning with a relative TASD equal to $22 \%$ in average we terminate the alignment with relative TASD $>95 \%$.

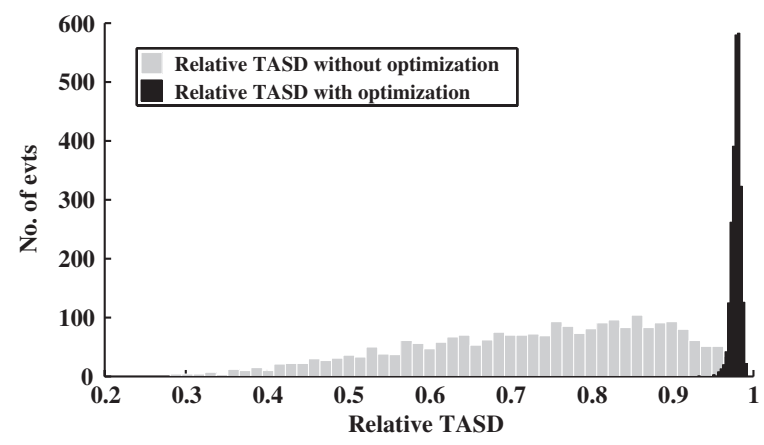

FIG. 16. Relative TASD results for $\xi_{2500}(3.8 \mu \mathrm{rad}, 20 \mu \mathrm{rad}$, $20 \mu \mathrm{m})$ simulated and optimized. The histogram in grey shows the relative TASD before the alignment procedure (SIMPLEX algorithm) and the black histogram shows the relative TASD reached after the optimization.

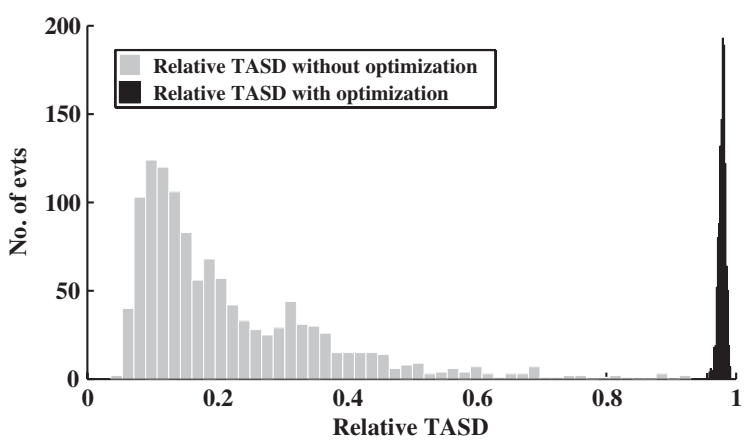

FIG. 17. Relative TASD results for $\xi_{1200}(3.8 \mu \mathrm{rad}, 100 \mu \mathrm{rad}$, $100 \mu \mathrm{m})$ simulated and optimized. The histogram in grey shows the relative TASD before the alignment procedure (SIMPLEX algorithm) and the black histogram shows the relative TASD reached after the optimization.
However, it is important to note that, in the end of the optimization procedure, the barycenter is located in average at $60 \mu \mathrm{m}$ away from the $z$ axis in the observation plane $z=0$ [instead of $14 \mu \mathrm{m}$ for the simulation set $\left.\xi_{2500}(3.8 \mu \mathrm{rad}, 20 \mu \mathrm{rad}, 20 \mu \mathrm{m})\right]$. This distance can be considered as reasonable since the electron axis can move in the transverse plane up to $80 \mu \mathrm{m}$ from the $z$ axis.

Finally, it is thus possible to optimize the TASD even in case of primary alignment being worse than expected. We can therefore consider that our method provides a good safety margin on the primary alignment tolerances.

\section{Laser and $\gamma$-ray beam polarizations}

With the waist and wavelength values of the recirculating Gaussian beam, one can safely assume the scalar paraxial approximation $[43,44]$ to describe the laser beam polarization transport. The Jones formalism [45] can then be used to compute the polarization changes induced by mirror reflections. Accordingly, the polarization vector $\mathbf{E}$ is perpendicular to the laser beam propagation axis and can be written as $\mathbf{E}_{n}=E_{s, n} \mathbf{s}_{n}+E_{p, n} \mathbf{p}_{n}$, where $\mathbf{s}_{n}$ and $\mathbf{p}_{n}$ are the perpendicular and parallel unit vectors to the plane of incidence of the $n$th reflection [45].

The mirrors' coating are made of dielectric quarter wave stacks [46]. When the laser beam incidence corresponds to the designed incident angle of the coatings, the $s$ and $p$ waves are dephased by $\pi$ and their reflection coefficients for the amplitude differ. However, with such coatings, and assuming stress-free mirror mounts, polarization ellipticity can be induced by angular misalignments [47] and by the very small birefringence that is currently measured in multilayer dielectric coatings [48].

We have computed the polarization transport in the recirculator taking into account its nonplanar geometry as described in [47]. We considered $\mathrm{SiO}_{2} / \mathrm{Ta}_{2} \mathrm{O}_{5}$ multilayer coatings with a transmittance of $\approx 500 \mathrm{ppm}$ (corresponding to 11 double layers) and we use the formalism of [49] to model it. All mirror reflections were taken into account, starting from the first $45^{\circ}$ injection mirror (i.e., mirror $M_{0}$ of Fig. 2). As for the parabolic reflectors and the MPS mirrors, we assumed coatings designed for $3.7^{\circ}$ and $22.5^{\circ}$ incident angle respectively. Since the laser beam incident angles are in fact $3.77^{\circ}$ and $22.54^{\circ}$ on these surfaces (see Table II), we thus introduce a slight polarization ellipticity after each mirror reflection.

The phasor representation [45], that is the real part of the complex field $\Re[\mathbf{E} \exp (i 2 \pi c t / \lambda)]$ in the fixed reference frame $\{x, y, z\}$ depicted in Fig. 1, is used to plot our results in Fig. 18(a) for all reflections. Writing the electric vector $\mathbf{E}_{\mathbf{l}}^{T}=\left(\cos \chi_{l} \sin \eta_{l}, \sin \chi_{l} \sin \eta_{l}, \cos \eta_{l}\right) \quad$ we obtain the angular distribution shown in Fig. 18(b) at the IP.

For these figures an incident $p$ wave was considered (i.e., $\mathbf{E}_{i n}=\mathbf{p}_{\text {in }}$, see Fig. 2) but similar results are obtained with the $s$ wave (i.e., $\mathbf{E}_{i n}=\mathbf{s}_{i n}$, see Fig. 2). Whereas large azimuthal angles are induced by the MPS reflections, 


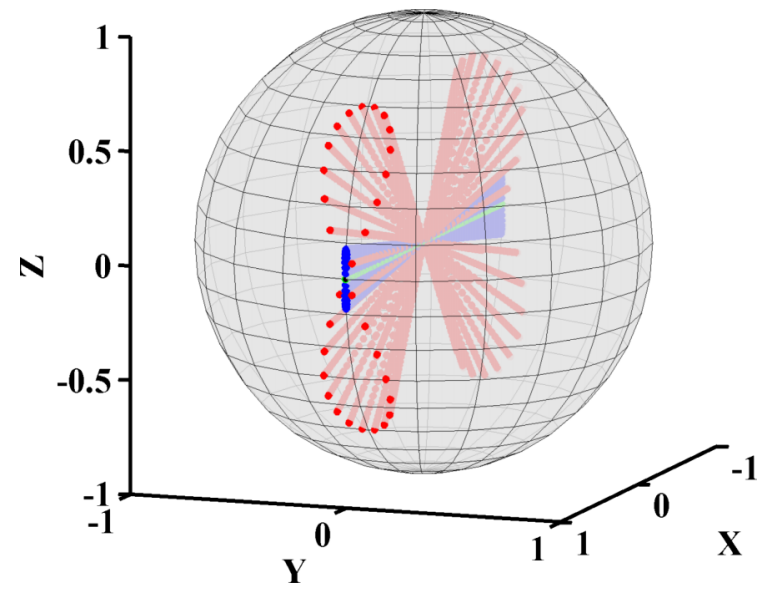

(a)

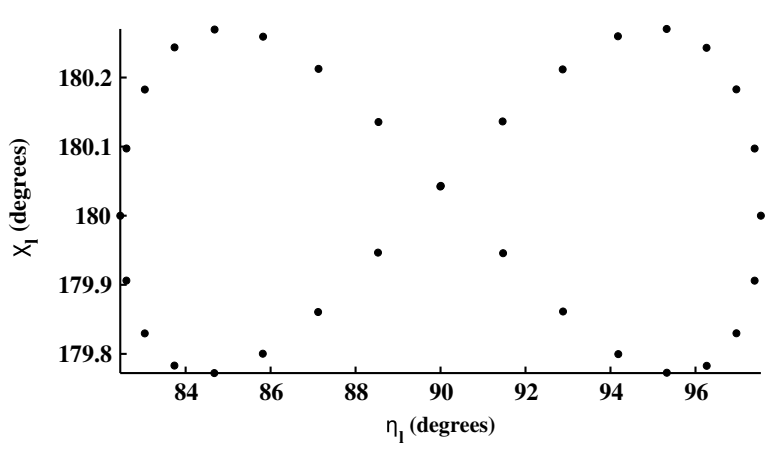

(b)

FIG. 18. (a) Phasor representation of the recirculating laser beam. In red the polarization after the reflection on the first mirror of the MPS, in blue the polarization at the IP, in black the polarization after the collimating parabolic mirror, and in green (over the black dots lying to the line of equation $y=z=0$ ) the polarization after the second mirror of the MPS. (b) Angular distribution of the azimuthal $\eta_{l}$ and polar $\chi_{l}$ angles at the IP corresponding to the blue circles of Fig. (a).

smaller variations of $\approx \pm 7.5^{\circ}$ of the polarization direction are observed at the IP. We checked our results using the CODE V software. Computing the normalized Stokes parameters $\left(S_{l 1}, S_{l 2}, S_{l 3}\right)$ [45] we obtain a degree of linear polarization [50] of the laser beam $\sqrt{S_{l 1}^{2}+S_{l 2}^{2}}=$ $1-4 \times 10^{-7}$ in average.

Misaligning randomly the recirculator as in Sec. VIC [i.e., $\xi_{5000}(3.8 \mu \mathrm{rad}, 20 \mu \mathrm{rad}, 20 \mu \mathrm{m})$ ] and adding a small coating birefringence as modeled in [48], we observed an average degradation of $\approx 0.3 \%$ for the degree of linear polarization (rms $0.2 \%$ ).

We also obtained similar results for other mirror coatings with high thermal damage threshold and large optical index, i.e., the currently used oxides $\mathrm{SiO}_{2} / \mathrm{ZrO}_{2}$ and $\mathrm{SiO}_{2} / \mathrm{HfO}_{2}$ [51].

In the end, the ensuing $\gamma$-ray beam polarization will be of great interest. From the laser beam polarization of Fig. 18(b), we calculate the $\gamma$-rays Stokes component

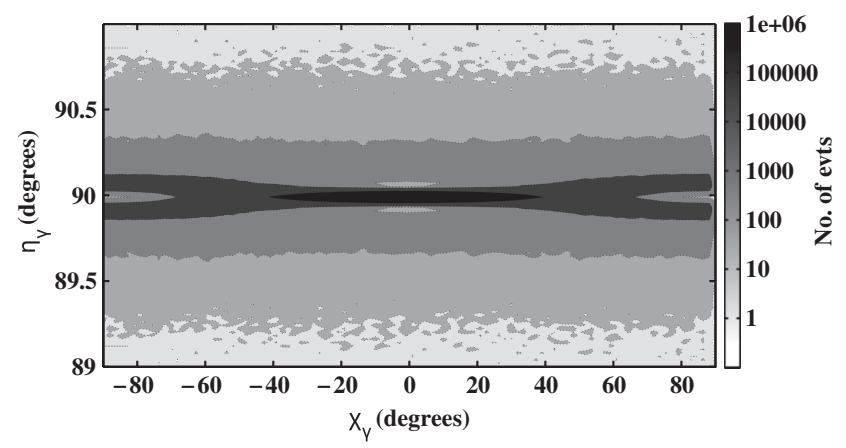

(a)

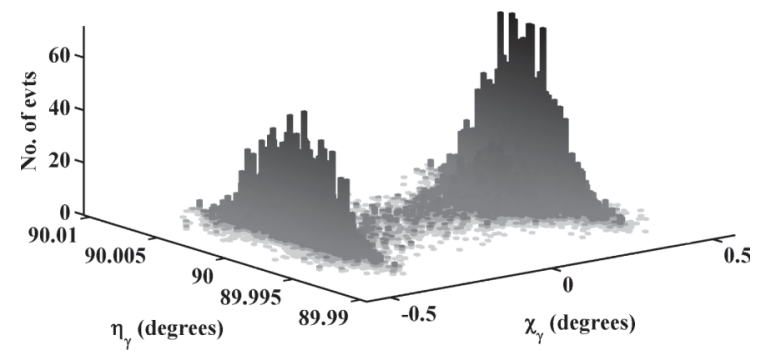

(b)

FIG. 19. (a) The angular distribution of the azimuthal angle $\eta_{\gamma}$ and polar angle $\chi_{\gamma}$ of the electric vector of the $\gamma$ rays produced by Compton interactions without energy selection. (b) The same angular distribution but for $\gamma$ rays energy above $0.99 E_{\max }$.

$\left(S_{\gamma 1}, S_{\gamma 2}, S_{\gamma 3}\right)$ [52] using the CAIN [36] simulation software. From the Stokes parameters and the direction of the $\gamma$ ray, we reconstructed the photon polarization vector [52]. As for the circulating laser beam, by writing $\mathbf{E}_{\gamma}{ }^{T}=$ $\left(\cos \chi_{\gamma} \sin \eta_{\gamma}, \sin \chi_{\gamma} \sin \eta_{\gamma}, \cos \eta_{\gamma}\right.$ ) we obtained the angular distributions of all simulated $\gamma$ rays shown in Fig. 19(a) and for those having energy ranged between $E_{\max }$ and $0.99 E_{\max }$ in Fig. 19(b) (with $E_{\max }$ the maximal energy of the scattered $\gamma$ rays). The high energy $\gamma$-ray therefore exhibits a very small angular dispersion of $\approx 1^{\circ}$ in $\chi_{\gamma}$ and $\approx 0.02^{\circ}$ in $\eta_{\gamma}$. As the degree of linear polarization from Stokes components, we obtain $>99.998 \%$. More generally, the closest to $E_{\max }$ is the energy of the $\gamma$ ray, the higher the degree of linear polarization.

\section{E. Constraints on mirror-surface quality}

The effect induced by a departure of the parabola shape from its design can be estimated by requiring a local slope variation below the tolerance level defined above, that is $\lesssim 1 \mu \mathrm{rad}$. With a laser beam radius on the optical surfaces $w_{M} \approx 8 \mathrm{~mm}$, we thus get $\approx 8 \mathrm{~nm}$ for the maximum departure value. This leads to a surface quality of $\approx \lambda / 60$ peak to valley which is nowadays available from high quality grade glass substrate polishers.

Another important issue related to the mirror surface shape is the matching of the parabola radii of curvatures. The simulations described above confirm the assumption of 
a circulating paraxial Gaussian beam that is aberration free. Accordingly, one can relate the laser beam waist at the IP of the $n$th pass $w_{0 n}$ to the first one by $w_{0 n} \approx\left(R_{1} / R_{2}\right)^{n-1} w_{01}$ [8], where $R_{i}$ is the radius of curvature of the $i$ th parabolic reflector. Eventually, we found that a relative difference below $0.1 \%$ between the two radii of curvatures is requested in order to keep the TASD losses below the percent level.

Finally, with the commercially available mirror coating transmittance of $500 \mathrm{ppm}$, we estimate the power losses due to reflections on optical surfaces to $\approx 2 \%$. This is the irreducible TASD loss of our system.

\section{CONCLUSION}

In this article, we have described a new kind of optical multipass device. It is dedicated to $\gamma$-ray production by Compton scattering between multibunch electron beams and of a high power laser for ELI-NP-GBS at the ELI-NP facility under development in Romania. A general method to design the recirculator's geometry and optical surfaces was developed for optimizing the $\gamma$-ray beam properties. Detailed numerical simulations were carried out within the framework of the specifications of the ELI-NP-GBS project.

Owing to the optical properties of the laser beam propagation inside the recirculator, we could set up a fast simulation code and thereafter show that the performance required by ELI-NP-GBS is achievable. Moreover, considering all the losses (due to synchronization, alignment, and mirror reflections) equal to $6 \%$, the recirculator has an enhancement factor of about 30 with respect to the TASD of a single pass.

However, the requested tolerances for the system alignment were found to be unrealistic with regard to standard optical alignment methods. To circumvent this problem a dedicated alignment procedure has been developed. It is simply based on the imaging of the position of the optical passes at the center of the recirculator. Moreover, we have demonstrated that this imaging system is also usable for synchronizing all optical passes with the electron bunches. Eventually, we implemented an alignment algorithm in our simulations starting from a rough optical alignment and ending up with the tight tolerance level required. We thus demonstrated that, in the case of the ELI-NP-GBS, an initial misalignment of the order of $100 \mu \mathrm{m}, 100 \mu \mathrm{rad}$ is acceptable.

On the other hand, our system contains a large number of high quality grade optical surfaces of which the properties have to be maintained. Therefore we have to take carefully into account the quality of the environment. In particular, severe conditions have to be respected for the cleanliness of the manufacturing process, during installation and during operation. In fact similar to the conditions to be respected for the National Ignition Facility and for Laser MegaJoule [53-55].

While the present recirculator has been designed for the ELI-NP-GBS project, it could also be used for other purposes. Examples are noncollinear optical parametric amplification [56] and high harmonic generation [57].

\section{APPENDIX A: CALCULATION OF THE PARAMETERS OF THE RECIRCULATOR}

Every optical and mechanical element constituting the recirculator will be manufactured with unavoidable tolerances on their sizes and radii of curvatures. However, since all the parameters are tightly linked together, these tolerances have to be taken into account in the geometrical design of the recirculator.

We thus define two sets of parameters. The first one contains the parameters externally imposed,

$$
\mathcal{E}_{\mathrm{ext}}=\left\{\lambda, U, F_{\max }, M^{2}, \tau, w_{0}, n_{d}, \phi, \nu_{r f}, \Delta \ell, \delta D_{\mathrm{MPS}}, e, D_{M}, \theta^{(0)}\right\},
$$

whereas the second one contains those parameters that have to be determined under the imposed external constraints,

$$
\mathcal{E}_{\text {opt }}=\left\{D, R_{C}, \Phi_{M}, N_{\text {pass }}, \theta, \theta_{\text {min }}, D_{\perp}, D_{\|}, D_{\text {str }}, D_{\mathrm{MPS}}, \ell\right\},
$$

where $\lambda, U, F_{\max }, M^{2}, \tau, w_{0}, n_{d}, \phi, D_{M}$ are defined in Sec. II $\mathrm{B} ; \nu_{r f}$ is the electron accelerating cavities rf; $\ell$ the optical path added by one MPS, and $\Delta \ell$ the corresponding variation range requested to tune the recirculation synchronization and to take into account the tolerances on the focal lengths of the parabolic mirrors (tolerance on $D$ ); $N_{\text {pass }}$ is the maximum possible number of passes; $D_{\perp}$ and $D_{\|}$the distances between the centers of two neighboring MPS in the $x y$ plane and along the $z$ axis respectively; $D_{\text {str }}$ the MPS mechanical stroke; $D_{\mathrm{MPS}}$ the distance between the mirrors of one MPS and $\delta D_{\text {MPS }}$ the manufacturing tolerance on this parameters; $e$ the mirror mounts thickness of MPS; $\theta^{(0)}$ is the input incident angle on the MPS, $\theta$ is the effective incident angle value which comes out after the procedure and $\theta_{\min }$ is the minimum $\theta$ angle that avoids laser beam vignetting induced by the MPS. In addition we define the notation $x^{(i)}$ that represents the intermediate value of $x$ determined at the step $i$, with $i=0$ the initial step.

In the calculations, the tolerances are taken into account by considering the worst case, it means that we must have 
the possibility to add $+\Delta \ell$ in the optical path with MPS delivered with a distance between mirrors equal to $D_{\text {MPS }}-\delta D_{\text {MPS }}$. Since some of the constraints are indeed tolerances, i.e., intervals, we have set up a mixed iterative and step by step procedure to determine $\mathcal{E}_{\text {opt }}$.

From Eqs. (1) and (2) we obtain $\Phi_{M}^{(0)}=2 n_{d} w_{M}$ and the initial parabolic mirrors distance $D^{(0)}=\frac{2 \pi w_{0}}{\lambda M^{2}} \sqrt{\frac{2 U}{\pi F_{\max }}}$; in addition from geometric consideration, Fig. 5 and assuming that $D_{\perp}=\Phi_{M}^{(0)}$, one can easily find the initial optical path $\ell^{(0)}=\Phi_{M}^{(0)} / \tan \theta^{(0)}$ added by the MPS. The recirculator round-trip must also be a harmonic $N_{r f}$ of the accelerating rf cavities so that the following expression holds:

$$
N_{r f}=\left\lceil\left(2 D^{(0)}+\ell^{(0)}\right) \nu_{r f} / c\right\rceil,
$$

where $\lceil x\rceil$ stands for the ceiling function of the real number $x$.

(a) First step: Parameters for synchronization.-In the first step of the procedure, the synchronization of the recirculator round-trip frequency and the accelerator if is taken into account. We determine the parameter set $\left\{D, \ell, R_{C}, \Phi_{M}\right\}$ which provides a synchronization between the recirculator and the electron cavities rf from the initial parameters. Taking into account the different mechanical tolerances, this set of constraints gives the following system of equations:

$$
\left\{\begin{array}{l}
\ell=2 D_{\mathrm{MPS}}^{(1)} \cos \theta^{(0)} \\
\ell+\Delta \ell=2\left(D_{\mathrm{MPS}}^{(1)}-\delta D_{\mathrm{MPS}}\right) \cos \theta_{\mathrm{min}}^{(1)} \\
\left(D_{\mathrm{MPS}}^{(1)}-\delta D_{\mathrm{MPS}}\right)=\frac{C_{1} D}{2 \sin \theta_{\min }^{(1)}} \\
2 D+\ell=L_{r f},
\end{array}\right.
$$

where $L_{r f}=N_{r f} c / \nu_{r f}$ is the length between two successive electron bunches and $C_{1}=n_{d} \lambda M^{2} / \pi w_{0}=\Phi_{M} / D$.

The parameters which come out from this system are

$$
\begin{gathered}
\theta_{\mathrm{min}}^{(1)}=\arccos \left(\frac{c}{\sqrt{a^{2}+b^{2}}}\right)-\arccos \left(\frac{a}{\sqrt{a^{2}+b^{2}}}\right), \\
D=\frac{\left(L_{r f}-2 \delta D_{\mathrm{MPS}} \cos \theta^{(0)}\right) \sin \theta_{\mathrm{min}}^{(1)}}{C_{1} \cos \theta^{(0)}+2 \sin \theta_{\mathrm{min}}^{(1)}} \\
\Phi_{M}=\frac{n_{d} \lambda M^{2} D}{\pi w_{0}}, \\
D_{\mathrm{MPS}}^{(1)}=\frac{\Phi_{M}}{2 \sin \theta_{\mathrm{min}}^{(1)}}+\delta D_{\mathrm{MPS}}, \\
\ell=2 D_{\mathrm{MPS}}^{(1)} \cos \theta^{(0)}, \\
D_{\perp}^{(1)}=\ell \tan \theta^{(0)},
\end{gathered}
$$

$$
R_{C}=D \tan \left(\frac{\phi}{2}\right)
$$

with

$$
\begin{gathered}
a=\left(C_{1} L_{r f}-2 C_{1} \delta D_{\mathrm{MPS}} \cos \theta^{(0)}\right), \\
b=-\left(4 \delta D_{\mathrm{MPS}} \cos \theta^{(0)}+2 \Delta \ell\right), \\
c=C_{1}\left(L_{r f}+\Delta \ell\right) \cos \theta^{(0)}
\end{gathered}
$$

(b) Second step: Mechanical stroke.-The second step computes primary values of the missing mechanical MPS parameters $\left\{D_{\text {str }}, D_{\|}\right\}$. These values are used in the next step to calculate the maximum number of passes and finally determined in the final step. We obtain from Figs. 5 and 20:

$$
\begin{gathered}
D_{\mathrm{str}}^{(2)}=R\left[\cos \left(\gamma+\theta_{\mathrm{min}}^{(1)}\right)-\cos \left(\theta^{(0)}+\gamma\right)\right], \\
D_{\|}^{(2)}=\frac{D_{\mathrm{MPS}}^{(1)} \cos \left(2 \theta^{(0)}\right)+D_{M}+2 D_{s t r}^{(2)}}{\cos \theta^{(0)}},
\end{gathered}
$$

where

$$
\begin{gathered}
A=\frac{D_{\mathrm{MPS}}^{(1)}}{\cos \theta^{(0)}}, \\
A_{\max }=\frac{D_{\mathrm{MPS}}^{(1)}}{\cos \theta_{\mathrm{min}}^{(1)}} \\
R=D_{\mathrm{MPS}}^{(1)}\left[\left(\sin \theta_{\mathrm{min}}^{(1)}+\sin \theta^{(0)}\right)^{2}\right. \\
\left.+\left(\frac{\cos \left(2 \theta^{(0)}\right)}{2 \cos \theta^{(0)}}-\sin \theta_{\min }^{(1)} \tan \theta^{(0)}\right)^{2}\right]^{\frac{1}{2}},
\end{gathered}
$$

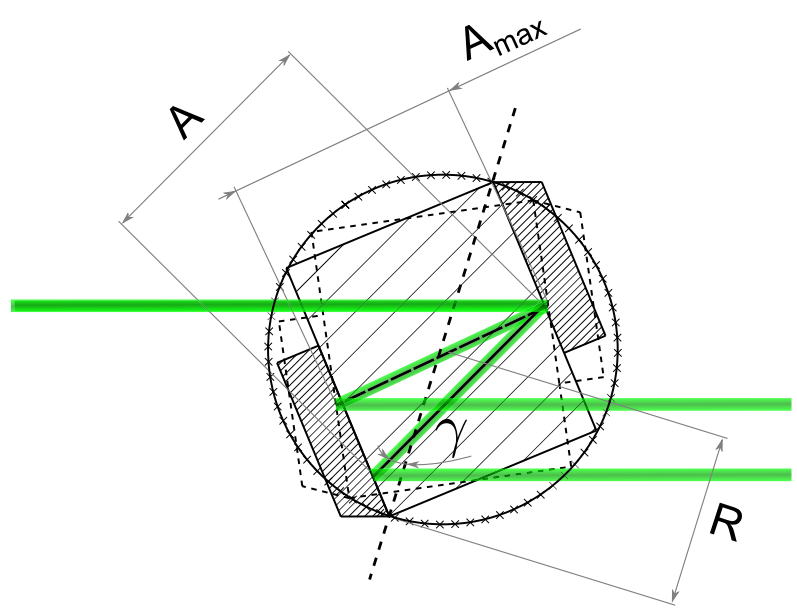

FIG. 20. Scheme of stroke calculation parameters. 


$$
\gamma=\arccos \left(\frac{A^{2}+R^{2}-\left(\frac{A_{\max }}{2}\right)^{2}}{2 A R}\right) .
$$

(c) Third step: Maximum number of passes available.We calculate, in this step, three number of passes, the first one $N_{\text {pass } 1}$ is the maximum number of laser beam spots possible on the circle of radius $R_{C}$, the second, $N_{\text {pass } 2}$ is the maximum number of passes possible under the rf constraint and $N_{\text {pass } 3}$ is the maximum possible number of MPS that can fit with the recirculator length (i.e., the length along the $z$ axis):

$$
\begin{gathered}
N_{\text {pass } 1}=4\left\lfloor\frac{\pi}{4 \arcsin \left(\frac{D_{\perp}^{(2)}}{2 R_{C}}\right)}\right\rfloor, \\
N_{\text {pass } 2}=4\left\lfloor\frac{\tau c}{4(2 D+\ell)}\right\rfloor, \\
N_{\text {pass 3 }}=4\left\lfloor\frac{L_{Z}}{4 D_{\|}^{(2)}}\right\rfloor,
\end{gathered}
$$

where $L_{Z}$ is the total length available along the $z$ axis for MPS and $\lfloor x\rfloor$ stands for the floor function of the real number $x$.

The maximum number of passes possible is then defined by $N_{\text {pass }}=\min \left(N_{\text {pass } 1} ; N_{\text {pass } 2} ; N_{\text {pass } 3}\right)$.

The procedure to compute $L_{Z}$ is developed in the following. We defined $z_{0}$ (see Fig. 21) as the solution of the equation of the variable $z$,

$$
\frac{\Phi_{M}}{2}+e+\frac{w(z)}{\cos \phi}-(L+w(z) \tan \phi-z) \sin \phi=0,
$$

where $w(z)=w_{0} \sqrt{1+\left[z \lambda M^{2} /\left(\pi w_{0}^{2}\right)\right]^{2}}$, the beam radius at the distance $z$ from the waist position and $L=$ $D /(1+\cos \phi)$ the propagation length from the parabolic reflector to the waist as shown in Fig. 21.

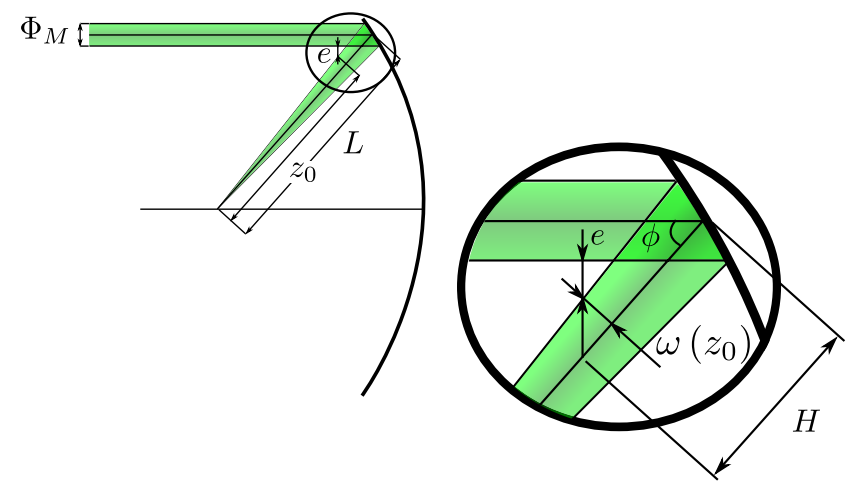

FIG. 21. Scheme of a reflection on a parabolic mirror.
Finally,

$$
L_{Z}=\frac{2 D \cos \phi}{1+\cos \phi}-2 H \cos \phi
$$

where $H=L+w\left(z_{0}\right) \tan \phi-z_{0}$ (see Fig. 21).

(d) Fourth step: $4 \pi$ matching.-Step four tunes the MPS parameters $D_{\text {MPS }}$ and $\theta$ to ensure a $4 \pi$ rotation of the laser pulse. This operation is done using the final value of $D_{\perp}$ which is defined by $N_{\text {pass }}$ and $R_{C}$. Then all parameters of the set $\mathcal{E}_{\text {opt }}$ are computed. In this section, we only give the equations to compute these MPS parameters, the reader can easily find these equations from Fig. 5 and setting the $\ell$ constant:

$$
\begin{gathered}
D_{\perp}=2 R_{C} \sin \left(\frac{\pi}{N_{\text {pass }}}\right), \\
\theta=\arctan \left(\frac{D_{\perp}}{\ell}\right), \\
D_{\mathrm{MPS}}=\frac{D_{\perp}}{2 \sin \theta} \\
\theta_{\min }=\arccos \left(\frac{\ell+\Delta \ell}{2\left(D_{\mathrm{MPS}}-\delta D_{\mathrm{MPS}}\right)}\right) .
\end{gathered}
$$

(e) Final step: Verification.-A final cross-check is performed to guarantee that all constraints are fulfilled for the value of $N_{\text {pass }}$ found in step three. These verifications are done on the maximum optical path length we can add up and the total size of occupancy along the $z$ axis of all the MPS. By defining $D_{\|}$as the new MPS occupation along the $z$ axis calculated as in step two using the latest parameters and $\Delta \ell^{(5)}=2\left(D_{\mathrm{MPS}}-\delta D_{\mathrm{MPS}}\right) \cos \theta_{\min }-\ell$ the new maximum length added to the optical path must obey to

$$
\begin{aligned}
& \Delta \ell^{(5)} \geq \Delta \ell, \\
& \left\lfloor\frac{L_{Z}}{D_{\|}}\right\rfloor \geq N_{\text {pass }} .
\end{aligned}
$$

Once these equations satisfied the computation is finished and the last parameters obtained in step four [i.e., the parameters without superscript $(i)]$ are the parameters taken for the recirculator design. If the equations are not satisfied one has to reduce $N_{\text {pass }}$ by four and iterate again the optimization at the fourth step.

\section{APPENDIX B: MPS PARALLELISM TUNING}

Here we succinctly describe the model for chain alignment of the MPS. In our simulations the calculations are done without approximation but the expressions derived 
below are very simple to use and exhibit important properties of the method.

\section{Model describing one MPS parallelism alignment}

The system is shown in Fig. 22 together with the reference axes and angles. We assume that mirror $M_{\text {ref }}$ has been aligned perpendicularly to $\mathbf{V}_{0}$ beforehand, i.e., $\mathbf{V}_{0}=-\mathbf{n}_{3}$. To describe the relative parallelism misalignment of the two mirrors, we introduce two angles $\varepsilon_{1} \ll 1$ and $\varphi_{1} \in[0,2 \pi]$ so that the normal vectors read $\mathbf{n}_{1}=-\mathbf{z}_{\mathbf{1}}$ and $\mathbf{n}_{2}=\varepsilon_{1} \cos \left(\varphi_{1}\right) \mathbf{x}_{\mathbf{1}}+\varepsilon_{1} \sin \left(\varphi_{1}\right) \mathbf{y}_{\mathbf{1}}+\mathbf{z}_{\mathbf{1}}+\mathbf{O}\left(\varepsilon_{1}^{2}\right)$. Note that we assume that $\varepsilon_{1}$ is small enough to justify first order calculations.

The vectors $\mathbf{V}_{0}$ and $\mathbf{V}^{\prime}{ }_{1}$ of Fig. 22 are related by $\mathbf{V}^{\prime}{ }_{1}=T U_{2} U_{1} \mathbf{V}_{0}$. In the basis $\left\{\mathbf{x}_{1}, \mathbf{y}_{1}, \mathbf{z}_{1}\right\}$, these matrices read as

$$
\begin{gathered}
U_{1}=\left[\begin{array}{ccc}
1 & 0 & 0 \\
0 & 1 & 0 \\
0 & 0 & -1
\end{array}\right], \\
U_{2}=\left[\begin{array}{ccc}
1 & 0 & -2 a \\
0 & 1 & -2 b \\
-2 a & -2 b & -1
\end{array}\right]+O\left(\varepsilon_{1}^{2}\right), \\
T=\left[\begin{array}{ccc}
1-2 \sin ^{2} \theta & 0 & -2 \sin \theta \cos \theta \\
0 & 1 & 0 \\
-2 \sin \theta \cos \theta & 0 & 1-2 \cos ^{2} \theta
\end{array}\right],
\end{gathered}
$$

where $a=\varepsilon_{1} \cos \varphi_{1}$ and $b=\varepsilon_{1} \sin \varphi_{1}$. Here $U_{1}$ describes a perfectly aligned mirror whereas the misalignment is entirely embodied in $U_{2}$. This is justified by the fact that the two successive reflections described by $U_{1}$ and $U_{2}$ are

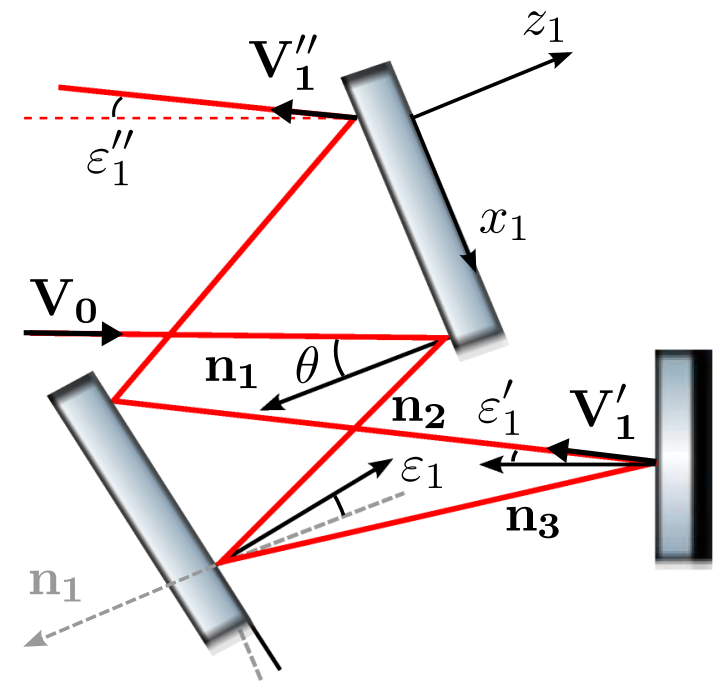

FIG. 22. Schematic view of misaligned first MPS with the main misalignment definition parameters. The coordinate system used in Appendix B is represented as well. equivalent to a rotation of angle $\varepsilon_{1}=2 \arccos \left(\mathbf{n}_{1} \cdot \mathbf{n}_{2}\right)$ around the direction $\mathbf{n}_{1} \times \mathbf{n}_{2}=\left(\sin \varphi_{1},-\cos \varphi_{1}, 0\right)^{T}$ whose matrix representation is indeed given by $U_{2} U_{1}$.

When adjusting the mirror pair parallelism, one measures the angles $\varepsilon_{1}^{\prime}$ and $\phi_{1}^{\prime}$ defined by

$$
\begin{gathered}
\varepsilon_{1}^{\prime}=\left\|\mathbf{V}_{1}^{\prime} \times \mathbf{n}_{3}\right\|, \\
\tan \varphi_{1}^{\prime}=\frac{\left(\mathbf{V}_{1}^{\prime} \times \mathbf{n}_{3}\right) \cdot \mathbf{y}_{1}}{\left(\mathbf{V}_{1}^{\prime} \times \mathbf{n}_{3}\right) \cdot \mathbf{V}_{0 \perp}} .
\end{gathered}
$$

This leads to the misalignment angles

$$
\begin{gathered}
\varepsilon_{1} \approx \varepsilon_{1}^{\prime} \frac{\sqrt{1-\sin ^{2} \theta \cos ^{2} \varphi_{1}^{\prime}}}{2 \cos \theta}, \\
\tan \varphi_{1} \approx \frac{\tan \varphi_{1}^{\prime}}{\cos \theta}
\end{gathered}
$$

When using an autocollimator we in fact measure the angle $\varepsilon_{1}^{\prime \prime}$ and $\varphi_{1}^{\prime \prime}$. The vectors $\mathbf{V}_{0}$ and $\mathbf{V}_{1}^{\prime \prime}$ are related by $\mathbf{V}_{1}^{\prime \prime}=U_{1} U_{2} T U_{2} U_{1} \mathbf{V}_{0}$. We obtain

$$
\begin{gathered}
\varepsilon_{1}^{\prime \prime}=\left\|\mathbf{V}_{1}^{\prime \prime} \times \mathbf{n}_{3}\right\|, \\
\tan \varphi_{1}^{\prime \prime}=\frac{\left(\mathbf{V}_{1}^{\prime \prime} \times \mathbf{n}_{3}\right) \cdot \mathbf{y}_{1}}{\left(\mathbf{V}_{1}^{\prime \prime} \times \mathbf{n}_{3}\right) \cdot \mathbf{V}_{0 \perp}},
\end{gathered}
$$

and thus

$$
\begin{gathered}
\varepsilon_{1} \approx \varepsilon_{1}^{\prime \prime} \frac{\sqrt{1-\sin ^{2} \theta \cos ^{2} \varphi_{1}^{\prime}}}{4 \cos \theta}, \\
\tan \varphi_{1} \approx \frac{\tan \varphi_{1}^{\prime \prime}}{\cos \theta} .
\end{gathered}
$$

The accuracy on the measurement of $\varepsilon_{1}$ becomes then just improved by a factor 2 .

Rotation transformation. - Let $R_{\mathbf{u}}(\beta)$ be the $3 \times 3$ matrix describing the rotation of angle $\beta$ around $\mathbf{u}$. We obtain $R_{\mathbf{u}}^{-1}(\beta) U_{2} U_{1} R_{\mathbf{u}}(\beta)=U_{2} U_{1}+O\left(\varepsilon_{1} \beta, \varepsilon_{1}^{2}, \beta^{2}\right)$. To the first order, $U_{2} U_{1}$ is thus invariant under rotation.

\section{Model for MPS parallelism chain alignment}

If we now chain $n$ MPS upstream $M_{1}$, we get

$$
\mathbf{V}_{n}{ }^{\prime}=T\left(\prod_{i=1}^{n} U_{2 i} U_{1 i}\right) \mathbf{V}_{0}
$$

with, to first order in $\varepsilon_{i}$,

$$
\prod_{i=1}^{n} U_{2 i} U_{1 i} \approx\left[\begin{array}{ccc}
1 & 0 & 2 A_{n} \\
0 & 1 & 2 B_{n} \\
-2 A_{n} & -2 B_{n} & 1
\end{array}\right],
$$


(a)

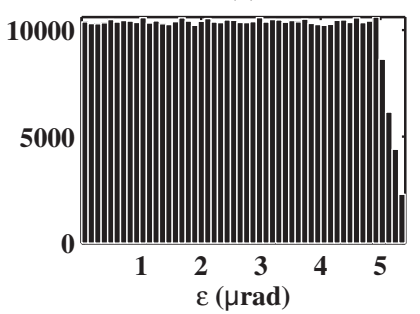

(c)

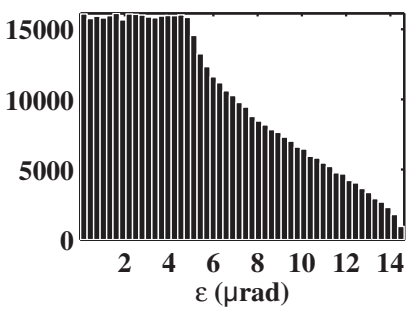

(b)

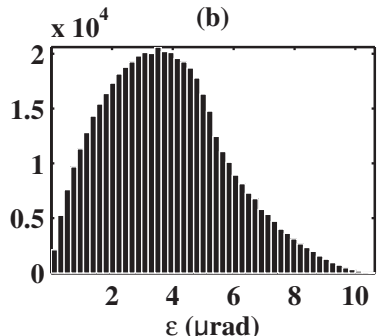

(d)

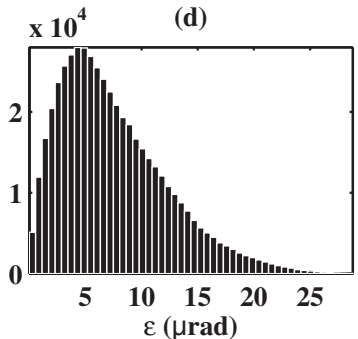

FIG. 23. Distributions of the misalignments of MPS (parallelism) $\varepsilon$ obtained with $\Delta \varepsilon^{\prime \prime}=20 \mu \mathrm{rad}$. (a) Of the first MPS for $\theta=22.5^{\circ}$. (b) Of the $n$th MPS, with $n>1$ for $\theta=22.5^{\circ}$. (c) Of the first MPS for $\theta=70^{\circ}$. (d) Of the $n$th MPS, with $n>1$ for $\theta=70^{\circ}$.

where $A_{n}=\sum_{i=1}^{n} \varepsilon_{i} \cos \varphi_{i}$ and $B_{n}=\sum_{i=1}^{n} \varepsilon_{i} \sin \varphi_{i}$. We then obtain the following recurrence relation which exhibits the correlations between the alignment steps:

$$
\begin{aligned}
\varepsilon_{n} \approx & \frac{1}{2 \cos \theta}\left(\cos ^{2} \theta\left(\varepsilon_{n}^{\prime} \cos \varphi_{n}^{\prime}-\varepsilon_{n-1}^{\prime} \cos \varphi_{n-1}^{\prime}\right)^{2}\right. \\
& \left.+\left(\varepsilon_{n}^{\prime} \sin \varphi_{n}^{\prime}-\varepsilon_{n-1}^{\prime} \sin \varphi_{n-1}^{\prime}\right)^{2}\right)^{1 / 2} \\
\tan \varphi_{n} \approx & \frac{\varepsilon_{n}^{\prime} \sin \varphi_{n}^{\prime}-\varepsilon_{n-1}^{\prime} \sin \varphi_{n-1}^{\prime}}{\varepsilon_{n}^{\prime} \cos \varphi_{n}^{\prime}-\varepsilon_{n-1}^{\prime} \cos \varphi_{n-1}^{\prime}} .
\end{aligned}
$$

The first equation shows that $\varepsilon_{n}$ increases with $\theta$ and that $\varepsilon_{1}$ (corresponding to the first MPS) is behaving differently than $\varepsilon_{n>1}$. This is illustrated by Fig. 23 where two extremes values of $\theta=22.5^{\circ}$ and $\theta=70^{\circ}$ have been considered. The distributions of $\varepsilon_{n}$ are the same for all $n>1$. For this figure, the angle $\varepsilon^{\prime \prime}$ was chosen randomly within the interval $\left[0, \Delta \varepsilon^{\prime \prime}\right]$ with $\Delta \varepsilon^{\prime \prime}=20 \mu \mathrm{rad}$. Finally, we have verified numerically that these expressions are accurate up to $\varepsilon^{\prime \prime} \simeq 1 \mathrm{mrad}$.

[1] P. Sprangle, A. Ting, E. Esarey, and A. Fisher, J. Appl. Phys. 72, 5032 (1992).

[2] E. Bulyak and V. Skomorokhov, in Proceedings of the 9th European Particle Accelerator Conference, Lucerne, 2004 (EPS-AG, Lucerne, 2004).

[3] P. Tomassini, A. Giulietti, D. Giulietti, and L. Gizzi, Appl. Phys. B 80, 419 (2005).

[4] P. Tomassini, A. Bacci, J. Cary, M. Ferrario, A. Giulietti, D. Giulietti, L. Gizzi, L. Labate, L. Serafini, V. Petrillo, and Vaccarezza, IEEE Trans. Plasma Sci. 36, 1782 (2008).

[5] V. Petrillo, A. Bacci, R. B. A. Zinati, I. Chaikovska, C. Curatolo, M. Ferrario, C. Maroli, C. Ronsivalle, A. Rossi, L. Serafini, P. Tomassini, C. Vaccarezza, and A. Variola, Nucl. Instrum. Methods Phys. Res., Sect. A 693, 109 (2012).

[6] A. Bacci et al., J. Appl. Phys. 113, 194508 (2013).

[7] O. Adriani et al., Technical Design Report, 2014.

[8] H. Kogelnick and T. Li, Appl. Opt. 5, 1550 (1966).

[9] T. Omori, T. Aoki, K. Dobashi, T. Hirose, Y. Kurihara, T. Okugi, I. Sakai, A. Tsunemi, J. Urakawa, M. Washio, and K. Yokoya, Nucl. Instrum. Methods Phys. Res., Sect. A 500, 232 (2003).

[10] A. Rollason, X. Fang, and D. Dugdale, Nucl. Instrum. Methods Phys. Res., Sect. A 526, 560 (2004).

[11] I. Yamane, in Proceedings of the International Particle Accelerator Conference, Kyoto, Japan (ICR, Kyoto, 2010).

[12] N. Falletto et al., Nucl. Instrum. Methods Phys. Res., Sect. A 459, 412 (2001).

[13] S. Baudrand et al., JINST 5, P06005 (2010).

[14] S. Miyoshi, T. Akagi, S. Araki, Y. Funahashi, T. Hirose, Y. Honda, M. Kuriki, X. Li, T. Okugi, T. Omori, G. Pei, K. Sakaue, H. Shimizu, T. Takahashi, N. Terunuma, J. Urakawa, Y. Ushio, and M. Washio, Nucl. Instrum. Methods Phys. Res., Sect. A 623, 576 (2010).

[15] J. Bonis, R. Chiche, R. Cizeron, M. Cohen, E. Cormier, P. Cornebise, N. Delerue, R. Flaminio, D. Jehanno, F. Labaye, M. Lacroix, R. Marie, B. Mercier, C. Michel, Y. Peinaud, L. Pinard, C. Prevost, V. Soskov, A. Variola, and F. Zomer, JINST 7, P01017 (2012).

[16] K. Sakaue, M. Washio, S. Araki, M. Fukuda, Y. Higashi, Y. Honda, T. Omori, T. Taniguchi, N. Terunuma, J. Urakawa, and N. Sasao, Rev. Sci. Instrum. 80, 123304 (2009).

[17] M. Y. Shverdin, I. Jovanovic, V. A. Semenov, S. M. Betts, C. Brown, D. J. Gibson, R. M. Shuttlesworth, F. V. Hartemann, C. W. Siders, and C. P. J. Barty, Opt. Lett. 35, 2224 (2010).

[18] I. Jovanovic, M. Shverdin, D. Gibson, and C. Brown, Nucl. Instrum. Methods Phys. Res., Sect. A 578, 160 (2007).

[19] J. Tümmler, R. Jung, H. Stiel, P. V. Nickles, and W. Sandner, Opt. Lett. 34, 1378 (2009).

[20] D. R. Herriott and H. J. Schulte, Appl. Opt. 4, 883 (1965).

[21] D. Kaur, A. M. de Souza, J. Wanna, S. A. Hammad, L. Mercorelli, and D. S. Perry, Appl. Opt. 29, 119 (1990).

[22] D. Korsch, Reflective Optics (Academic Press, New York, 1991).

[23] A. E. Siegman, Proc. SPIE Int. Soc. Opt. Eng. 1868, 2 (1993).

[24] J. Marioge, J. Opt. 24, 177 (1993).

[25] J. Marioge, J. Opt. 24, 217 (1993).

[26] N. Bloembergen, Appl. Opt. 12, 661 (1973).

[27] R. Bonnand, J. Degallaix, R. Flaminio, L. Giacobone, B. Lagrange, F. Marion, C. Michel, B. Mours, P. Mugnier, E. Pacaud, and L. Pinard, Classical Quantum Gravity 30, 155014 (2013).

[28] W. J. Brown and F. V. Hartemann, Phys. Rev. ST Accel. Beams 7, 060703 (2004). 
[29] C. Maroli, V. Petrillo, P. Tomassini, and L. Serafini, Phys. Rev. ST Accel. Beams 16, 030706 (2013).

[30] B. Badelek et al., Int. J. Mod. Phys. A 19, 5097 (2004).

[31] G. Moortgat-Pick et al., Phys. Rep. 460, 131 (2008).

[32] B. A. Reagan, K. A. Wernsing, A. H. Curtis, F. J. Furch, B. M. Luther, D. Patel, C. S. Menoni, and J. J. Rocca, Opt. Lett. 37, 3624 (2012).

[33] B. C. Stuart, M. D. Feit, S. Herman, A. M. Rubenchik, B. W. Shore, and M. D. Perry, Phys. Rev. B 53, 1749 (1996).

[34] E. Hetch, Optics (Addison-Wesley, Incorporated, 2002), p. 426.

[35] U. Fano, J. Opt. Soc. Am. 39, 859 (1949).

[36] K. Yokoya, "A computer simulation code for the interaction of electron, positron, gamma beams and strong lasers," http://lcdev.kek.jp/ yokoya/CAIN/.

[37] Code V, version 10.5 (Synopsys Inc., 3280 East Foothill Boulevard, Suite 300 Pasadena, California 91107-3103, USA, 2012).

[38] B. Suzuki, KEK note kek-76-3, 1976.

[39] MATLAB, version 8.0.0.783 (R2012b) (The MathWorks Inc., Natick, Massachusetts, 2012).

[40] C. Bond and C. A. Pipan, Proc. SPIE Int. Soc. Opt. Eng. 1113, 236 (1989).

[41] D.-C. Chen, Int. J. Jpn. Soc. Precision Eng. 13, 33 (2012).

[42] J. Lagarias, J. Reeds, M. Wright, and P. Wright, SIAM J. Optim. 9, 112 (1998).

[43] P. Varga and P. Török, Opt. Commun. 152, 108 (1998).

[44] F. Zomer, J. Opt. Soc. Am. A 20, 172 (2003).
[45] R. M. Azzam and N. M. Bashara, Ellipsometry and Polarized Light (Elsevier, New York, 1987).

[46] C. J. Hood, H. Kimble, and J. Ye, Phys. Rev. A 64, 033804 (2001).

[47] F. Zomer, Y. Fedala, N. Pavloff, V. Soskov, and A. Variola, Appl. Opt. 48, 6651 (2009).

[48] F. Bielsa, A. Dupays, M. Fouché, R. Battesti, C. Robilliard, and C. Rizzo, Appl. Phys. B 97, 457 (2009).

[49] P. Yeh, J. Opt. Soc. Am. 69, 742 (1979).

[50] D. P. Barber, H.-D. Bremer, M. Böge, R. Brinkmann, W. Brückner, C. Büscher, M. Chapman, K. Coulter, P. Delheij, M. Düren et al., Nucl. Instrum. Methods Phys. Res., Sect. A 329, 79 (1993).

[51] B. Mangote, Ph.D. thesis, Université Paul CézanneAix-Marseille III, 2011.

[52] V.B. Berestetskij, Course of Theoretical Physics: Quantum Electrodynamics (Butterworth-Heinemann, Washington, DC, 1982), Vol. 4.

[53] F. Y. Génin, M. D. Feit, M. R. Kozlowski, A. M. Rubenchik, A. Salleo, and J. Yoshiyama, Appl. Opt. 39, 3654 (2000).

[54] S. Palmier, Ph.D. thesis, Université Sciences et Technologies-Bordeaux I, 2007.

[55] K. Bien-Aimé, Ph.D. thesis, Université Sciences et Technologies-Bordeaux I, 2009.

[56] B. Trophème, B. Boulanger, and G. Mennerat, Opt. Express 20, 26176 (2012).

[57] S. Fomichev, P. Breger, B. Carre, P. Agostini, and Zaretsky, Laser Phys. 12, 383 (2002). 